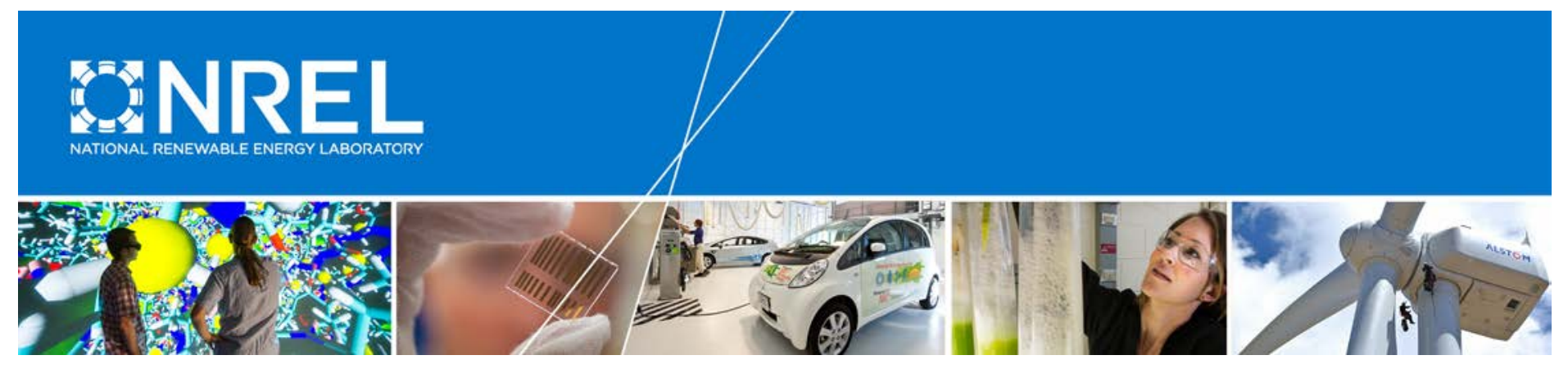

\title{
Cross-Border Electricity Trade between India and Sri Lanka: Impact on Power System Operations
}

Amy Rose, Brendan McBennett, David Palchak, and Jaquelin Cochran National Renewable Energy Laboratory

H.M. Wijekoon, Buddhika Samarasekara, and Randika Wijekoon

Ceylon Electricity Board

NREL is a national laboratory of the U.S. Department of Energy Office of Energy Efficiency \& Renewable Energy Operated by the Alliance for Sustainable Energy, LLC

This report is available at no cost from the National Renewable Energy Laboratory (NREL) at www.nrel.gov/publications.

Technical Report

NREL/TP-6A20-71983

December 2018 


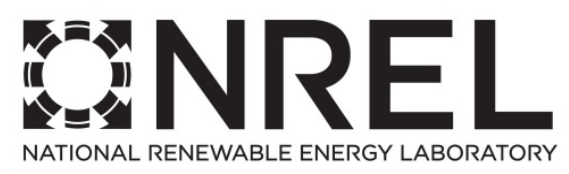

\title{
Cross-Border Electricity Trade between India and Sri Lanka: Impact on Power System Operations
}

\author{
Amy Rose, Brendan McBennett, \\ David Palchak, and Jaquelin Cochran \\ National Renewable Energy Laboratory \\ H.M. Wijekoon, Buddhika Samarasekara, \\ and Randika Wijekoon \\ Ceylon Electricity Board
}

\section{Suggested Citation}

Rose, Amy, Brendan McBennett, David Palchak, Jaquelin Cochran, H.M. Wijekoon, Buddhika Samarasekara, Randika Wijekoon. 2018. CrossBorder Electricity Trade between India and Sri Lanka: Impact on Power System Operations. Golden, CO: National Renewable Energy Laboratory. NREL/TP-6A20-71983. https://www.nrel.gov/docs/fy19osti/71983.pdf.

NREL is a national laboratory of the U.S. Department of Energy Office of Energy Efficiency \& Renewable Energy Operated by the Alliance for Sustainable Energy, LLC

This report is available at no cost from the National Renewable Energy Laboratory (NREL) at www.nrel.gov/publications.

National Renewable Energy Laboratory 15013 Denver West Parkway Golden, CO 80401

303-275-3000 • www.nrel.gov

\section{Technical Report}

NREL/TP-6A20-71983

December 2018

Contract No. DE-AC36-08GO28308 


\section{NOTICE}

This work was authored, in part, by the National Renewable Energy Laboratory, operated by Alliance for Sustainable Energy, LLC, for the U.S. Department of Energy (DOE) under Contract No. DE-AC36-08G028308. Funding provided by the U.S. Department of State's Bureau of South and Central Asian Affairs under Agreement 1931CQ16Y0002-160002. The views expressed in this publication do not necessarily represent the views of the DOE or the U.S. Government including Department of State.

This report is available at no cost from the National Renewable Energy Laboratory (NREL) at www.nrel.gov/publications.

U.S. Department of Energy (DOE) reports produced after 1991 and a growing number of pre-1991 documents are available free via www.OSTI.gov.

Cover Photos by Dennis Schroeder: (left to right) NREL 26173, NREL 18302, NREL 19758, NREL 29642, NREL 19795.

NREL prints on paper that contains recycled content. 


\section{Abstract}

Recent developments in South Asia, including rapid expansion of wind and solar generation, have the potential to increase the incentives for cross-border energy trade (CBET) between India and Sri Lanka. This study builds on several prior analyses of a potential high-voltage direct current transmission link between India and Sri Lanka. Uniquely, the study makes use of a detailed one-year, 30-minute resolution unit commitment and economic dispatch (UC\&ED) model of both countries to examine the technical and economic impacts of CBET between India and Sri Lanka in the year 2025. The study finds that CBET, enabled by a 500-MW high voltage direct current transmission link, could generate annual production cost savings of USD 180 million and improve power system operations. Generation from relatively expensive natural gas plants in Sri Lanka is displaced by lower cost imports from India, enabling fuel savings through better utilization of more cost-effective generators. In addition, increased flexibility through exports reduces RE curtailment in India's Southern region by $8.5 \%$, or $400 \mathrm{GWh}$, annually. While the study captures many previously unconsidered intertemporal operational constraints, it does not provide a complete characterization of system operations with a high-voltage direct current interconnection. Additional work should include a dynamic analysis of system stability following interruptions to the direct current interconnection, an AC power flow analysis and consideration of any non-technical barriers to trade which might prevent the optimization of CBET between India and Sri Lanka at 30-minute timescales.

\section{Acknowledgments}

The authors are greatly indebted to the thoughtful feedback we have received throughout the project, including from Jeff Ficken, Olivier Garaud, and Partha Mazumdar, U.S. Department of State; Monali Hazra and Priya Sreedharan, U.S. Agency for International Development; Auxilliam Jayamary and M. Rajagopalan, Tamil Nadu Transmission Corporation Limited; Manish Keshari and Narendra Sathvik, POWERGRID; Ishan Sharan, Central Electricity Authority; and the participants at South Asia Regional Initiative for Energy Integration (SARI/EI) workshop in Sri Lanka in March 2018. We wish to also thank at NREL Dan Bilello, Ilya Chernyakhovskiy, and Jennie Jorgenson for their reviews and Justin Rickard for the technical edit to this paper. Any errors or omissions are solely the responsibility of the authors.

This analysis was funded by the U.S. Department of State's Bureau of South and Central Asian Affairs Regional Connectivity Program. 


\section{List of Acronyms}

CBET

CEB

ER

GJ

GW

GWh

INR

IPP

IRADe

MW

MWh

NER

NR

NREL

PLF

$\mathrm{PSS} / \mathrm{E}$

RE

SAM

SARI/EI

SR

TWh

UC\&ED

USD

WR cross-border energy trade

Ceylon Electricity Board

Eastern region

gigajoule

gigawatt

gigawatt hour

India rupee

independent power producer

Integration Research and Action for Development

megawatt

megawatt hour

Northeastern region

Northern region

National Renewable Energy Laboratory

plant load factor

Power System Simulator for Engineering

renewable energy

System Advisor Model

South Asia Regional Initiative for Energy Integration

Southern region

terawatt hour

unit commitment and economic dispatch

United States dollar

Western region 


\section{Executive Summary}

Recent developments in South Asia have the potential to increase the incentives for cross-border energy trade (CBET) between India and Sri Lanka. India's surplus power, including from its rapid expansion of wind and solar generation, provides an incentive for it to increase investment returns through exports. Sri Lanka is considering options to increase its power supply, and CBET could reduce the need for investments in new thermal generation capacity, while giving Sri Lanka access to India's large and diverse power system. Finally, CBET can facilitate renewable energy (RE) integration by allowing the two countries to more efficiently harness balancing resources and smooth out wind and solar variability over larger geographic areas.

This study builds on several prior analyses of a potential high-voltage direct current transmission link between India and Sri Lanka. Uniquely, the study makes use of a detailed one-year, 30minute resolution unit commitment and economic dispatch (UC\&ED) model of both countries to examine the technical and economic impacts of CBET between India and Sri Lanka in the year 2025. This study finds that CBET, enabled by a 500-MW high voltage direct current transmission link, between India and Sri Lanka in 2025 presents an opportunity to reduce the cost of providing electricity, reduce RE curtailment in southern India, and improve system reliability through coordinated use of energy resources.

\section{Cost of Generating Electricity Decreases USD 180 Million Annually}

A direct current (DC) tie in 2025 saves USD 180 million annually in production costs, a $0.5 \%$ decrease in total cost of generating electricity in the two countries. The cost savings occur in Sri Lanka, where annual production costs decrease 35\%. Production costs remain almost unchanged in India, where cross-border trade represents a small fraction of overall generation.

\section{Imports from India displace over 69\% of Sri Lanka's natural gas generation}

The annual cost savings occur because the DC tie allows for a more efficient use of available generation between the two systems. In India, exports increase coal generation in Southern, Western, and Eastern regions and RE generation (from reduced RE curtailment) in the Southern region. In Sri Lanka, imports from India increase available power, allowing Sri Lanka to shift more of its daytime large hydro generation to evening to meet its peak load. Combined, these factors decrease the need for peaking generation in Sri Lanka, including natural gas generation, which falls $70 \%$ annually. Natural gas accounts for $16 \%$ of the country's generation without a tie.

\section{Sri Lanka exports to India $14 \%$ of the year}

Energy exchanges are bidirectional. It is less expensive to import power from Sri Lanka to India for $14 \%$ of the year, primarily December, when high hydro and coal generation in Sri Lanka creates export opportunities to India. The DC tie is congested for $58 \%$ of the year, implying that transfer capacity beyond $500 \mathrm{MW}$ could provide additional economic benefits through trade in both directions.

\section{Daytime exports from India to Sri Lanka reduce RE curtailment in India's Southern} region by $9 \%$

India's Southern region is more susceptible to RE curtailment (wind and solar energy that is available but not used) compared to other regions in India (Palchak et al. 2017). A DC tie to Sri 
Lanka provides an avenue for energy exports during times when solar and wind generation is high and thermal generation cannot be turned down. The DC tie reduces annual RE curtailment in the Southern region by $9 \%$ (400 gigawatt hours).

Reliability in Sri Lanka improves through coordinated use of energy resources

Interannual variability in hydropower resources creates a security of supply risk for Sri Lanka's power system. Cross-border trade reduces this risk by increasing the availability of reserves from generation sources in India. However, the DC tie also creates potential operational and national security risks, including that the DC tie serves $12 \%$ of Sri Lanka's annual load. While the DC tie can help address contingencies in Sri Lanka, this service is dependent on a coordinated response between the countries.

While the study captures many previously unconsidered intertemporal operational constraints, it does not provide a complete characterization of system operations with a high-voltage direct current interconnection. Additional work should include a dynamic analysis of system stability following interruptions to the direct current interconnection, an $\mathrm{AC}$ power flow analysis and consideration of any non-technical barriers to trade which might prevent the optimization of CBET between India and Sri Lanka at 30-minute timescales. 


\section{Table of Contents}

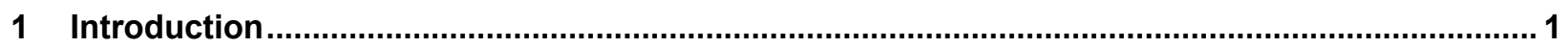

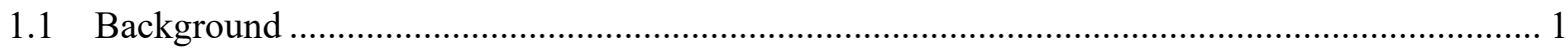

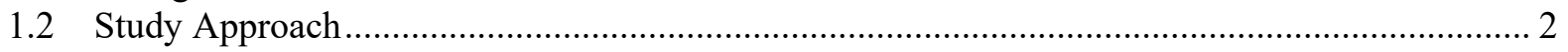

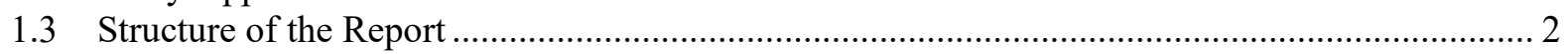

2 Study Scenarios, Modeling Methodology, and Assumptions................................................. 3

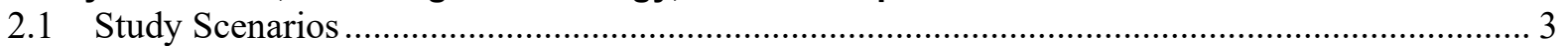

2.2 Unit Commitment and Economic Dispatch Model ............................................................ 4

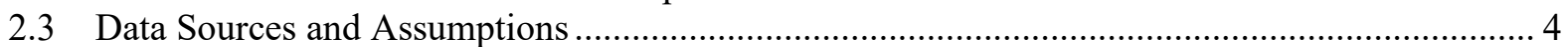

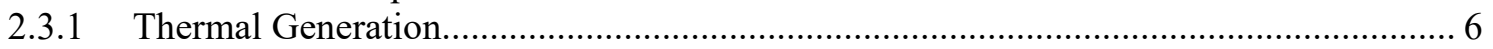

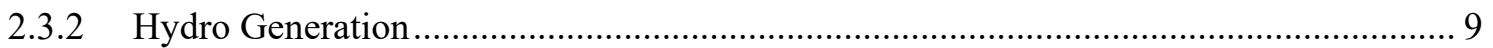

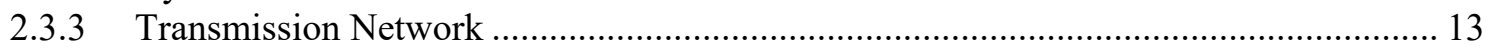

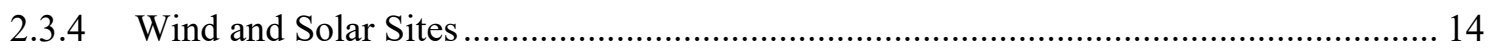

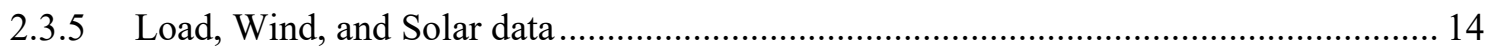

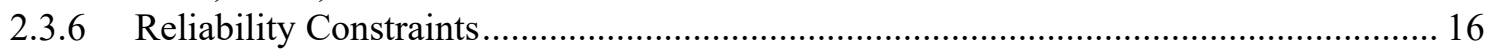

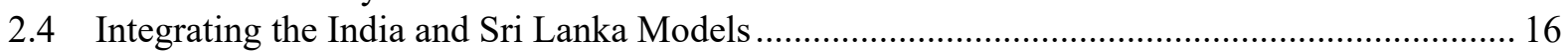

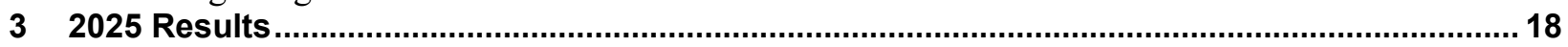

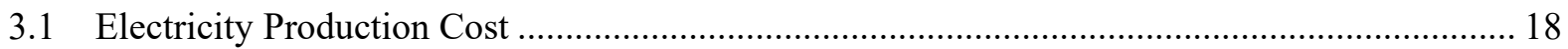

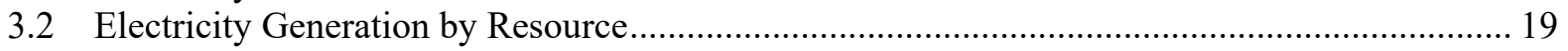

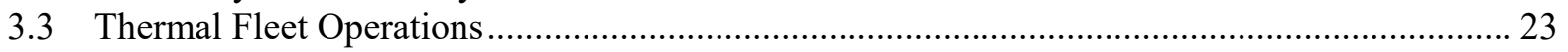

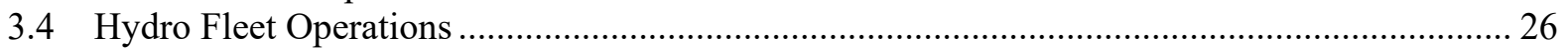

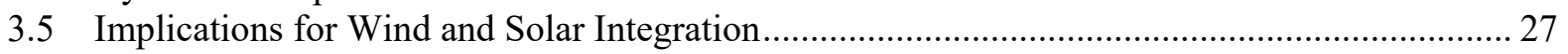

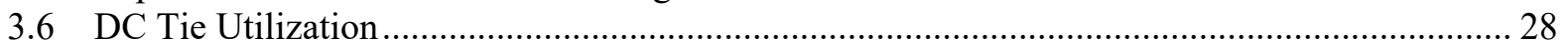

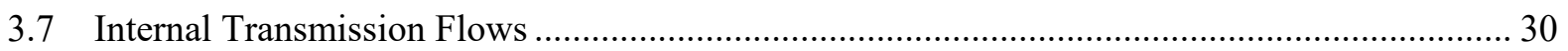

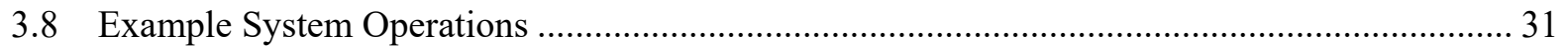

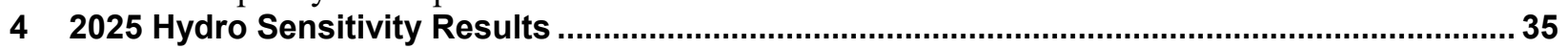

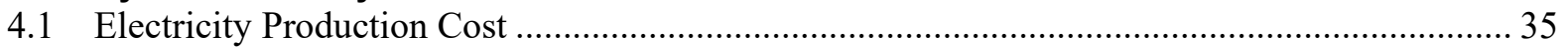

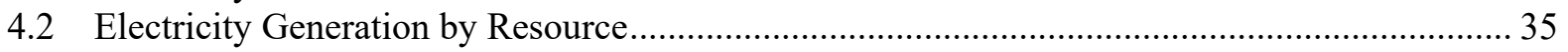

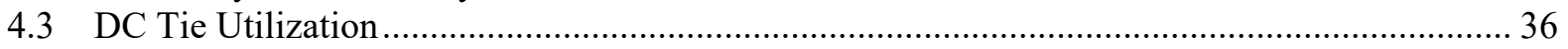

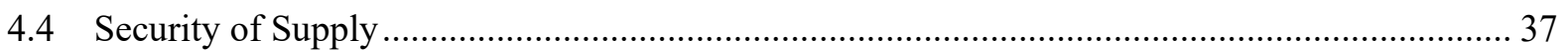

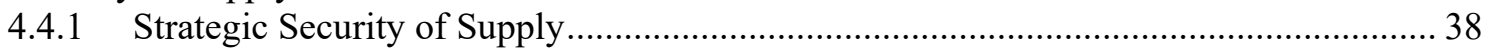

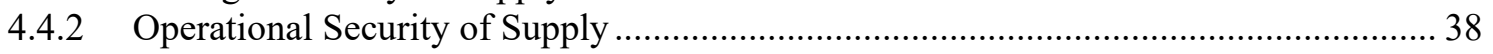

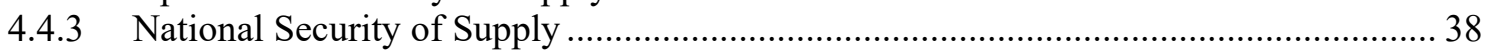

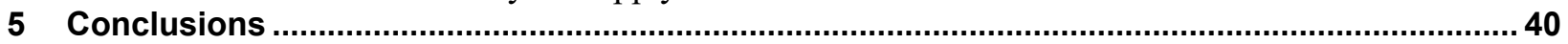

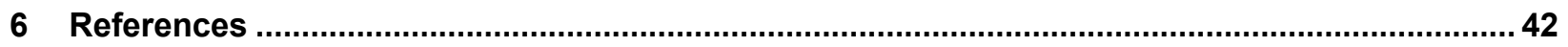

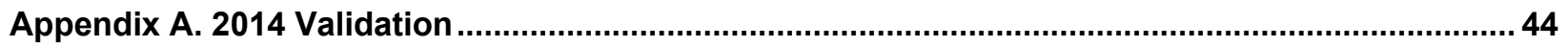




\section{List of Figures}

Figure 1. Process for building and simulating the 2025 Sri Lanka power system ................................... 5

Figure 2. Comparison of installed capacity by resource type in the 2014 and 2025 Sri Lanka databases.... 6

Figure 3. 2025 merit order for Sri Lanka's thermal generators operating at max capacity ....................... 9

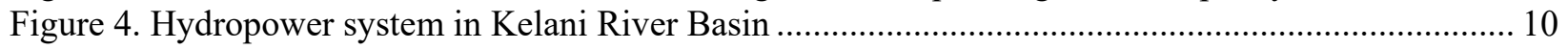

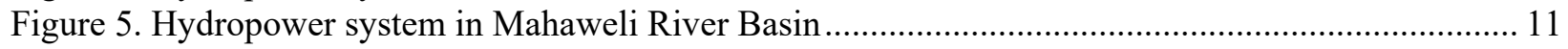

Figure 6. Comparison of Sri Lanka's average hourly available wind, solar, and mini-hydro energy and

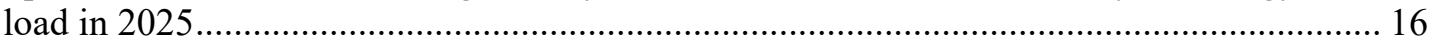

Figure 7. Generation and plant load factor by resource type in Sri Lanka, with and without a DC tie..... 20

Figure 8. Change in generation by resource type with a DC tie, 4 India regions and Sri Lanka............... 21

Figure 9. Comparison by month of (A) Sri Lanka's hydro availability, (B) Sri Lanka's change in generation with a DC tie, and (C) production cost savings across both Sri Lanka and India

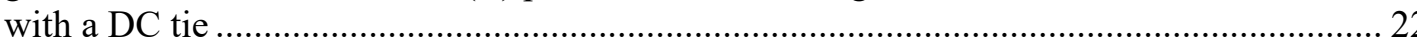

Figure 10. Coal generator operations with and without a DC tie, Sri Lanka......................................... 24

Figure 11. Other thermal generator operations with and without a DC tie, Sri Lanka ............................ 25

Figure 12. Hourly net load ramp rates as a ratio of non-RE online capacity: Southern region (SR), Sri Lanka and the combined SR + Sri Lanka footprint created by the DC tie............................26

Figure 13. Sri Lanka's average hourly large hydro generation (top panel) and average hourly pumped storage operations (bottom panel), with and without a DC tie

Figure 14. Comparison of total annual intertie flows and RE curtailment reduction in India with a DC tie,

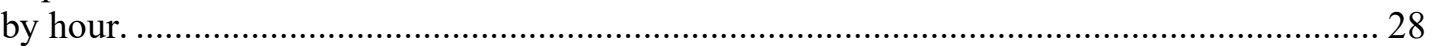

Figure 15. Duration curve of (A) DC tie flows and (B) DC tie flows normalized to Sri Lanka's load. ..... 29

Figure 16. Annual energy flows on Sri Lanka's transmission network with and without the DC tie......... 30

Figure 17. Sri Lanka's April 10 dispatch, with and without the DC tie.................................................. 31

Figure 18. Sri Lanka's November 30 dispatch, with and without the DC tie ........................................ 32

Figure 19. Dispatch in Sri Lanka and Tamil Nadu on June 14, illustrating:........................................... 33

Figure 20. Change in generation with the DC tie by resource type and region for each hydro sensitivity. 36

Figure 21. Daily and seasonal patterns of trade between India and Sri Lanka for all hydro sensitivities... 37

Figure 22. Fraction of annual load met by imports every period in Sri Lanka ........................................ 39

Figure 23. Comparison of Sri Lanka's actual and modeled 2014 generation by (A) resource and (B)

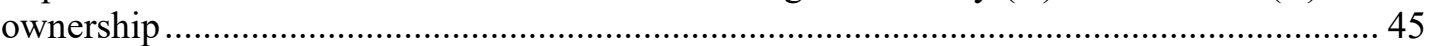

Figure 24. Comparison of Sri Lanka's actual and modeled 2014 CEB-owned hydro generation .............. 46

Figure 25. Comparison of actual and modeled 2014 monthly generation at six of Sri Lanka's major

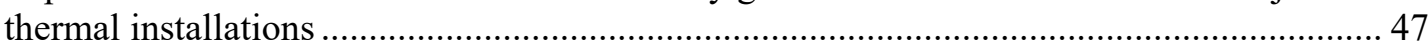

\section{List of Tables}

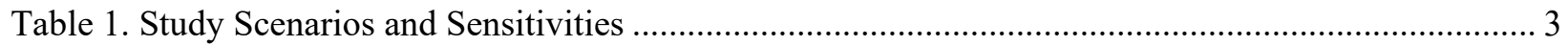

Table 2. Monthly Scalars for Wet and Dry Hydro Years in Sri Lanka .................................................. 3

Table 3. Average CEB 2025 Thermal Parameters .......................................................................... 7

Table 4. Average IPP 2025 Thermal Parameters ...................................................................... 8

Table 5. Sri Lanka Hydro Generator Parameters and Operating Constraints ....................................... 12

Table 6. Basic Information on Modeled 2025 Sri Lanka Transmission Network .................................... 13

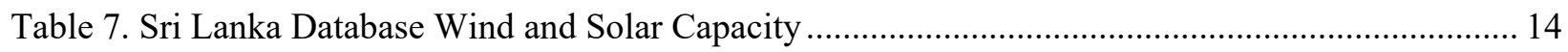

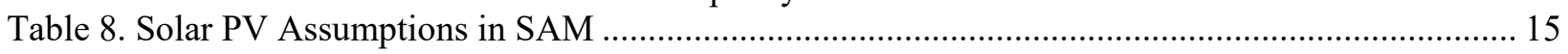

Table 9. Conversions Applied When Integrating the India and Sri Lanka Models into a Combined 'South

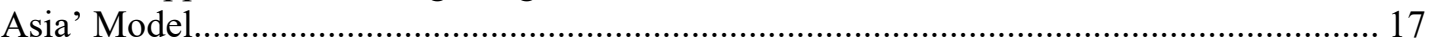

Table 10. Total (\$M) and Per-Unit (\$/MWh) Electricity Production Cost, with and without DC Tie, India

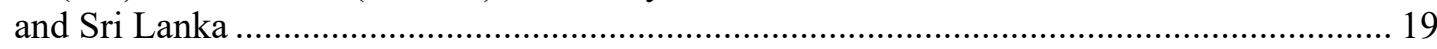


Table 11. Summary of Coal Fleet Operations in India and Sri Lanka without and with DC Tie (No DC / DC), Capacity Weighted Averages ........................................................................... 23

Table 12. Summary of Other Thermal Operations in India and Sri Lanka with and without DC Tie (No

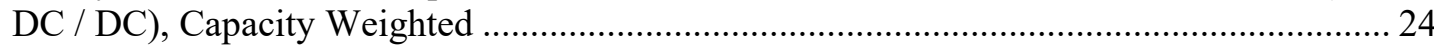

Table 13. RE Curtailment in India and Sri Lanka, with and without DC Tie........................................ 28

Table 14. Electricity Production Cost with and without DC Tie, India and Sri Lanka, Hydro Sensitivities 35

Table 15. Annual Energy Imports (TWh) and Fraction of Annual Load Met by Imports (\%) in Each Hydro Sensitivity 39 


\section{Introduction}

Recent developments in the South Asia region have the potential to increase the incentives for cross-border energy trade (CBET) between India and Sri Lanka. First, India is projected to have surplus power in most regions of the country by the mid-2020s (CEA, 2018). This provides an incentive for India to increase return on investments through trade while also presenting an opportunity for neighboring countries to gain access to a large power system diverse energy resources. Second, Sri Lanka's power system planners are evaluating options for new sources of supply to address rising demand. Imports through CBET could increase available supply and potentially avoid the need for investments in new thermal generation capacity. Third, falling technology costs and national energy policies have led to significant growth in renewable energy (RE) investments in the region, with much more expected in the coming years. Coordinated regional trade can facilitate RE integration by leveraging the geographic diversity of weather over a broader area to reduce variability of wind and solar generation and by increasing access to resources available for balancing (Palchak et al. 2017a). This study explores the impacts of a transmission connection between India and Sri Lanka on technical and economic aspects of operations on both systems in the year 2025 .

\subsection{Background}

India has an installed generation capacity of 331 gigawatts $(\mathrm{GW})$, with coal and renewable energy sources accounting for $193 \mathrm{GW}(58 \%)$ and $63 \mathrm{GW}(19 \%)$, respectively, and a peak demand of $164 \mathrm{GW}$ (CEA 2017b). The Government of India has set a target of $175 \mathrm{GW}$ of RE by 2022, including $100 \mathrm{GW}$ of solar and $60 \mathrm{GW}$ of wind. India anticipates its peak demand to grow to $230 \mathrm{GW}$ by 2022 (CEA 2017a).

Sri Lanka's power system differs significantly from that of India in both size and composition. The annual peak demand is $2.3 \mathrm{GW}$ and is expected to reach $3.8 \mathrm{GW}$ by 2025. Sri Lanka's installed capacity is $3.8 \mathrm{GW}$ and heavily reliant on rainfall, with hydropower accounting for $44 \%$ of installed capacity and 46\% of annual energy generation in 2015 (CEB 2015). The Government of Sri Lanka has set a goal of $20 \%$ energy from non-conventional renewable energy-i.e., minihydro, biomass, wind, and solar-by 2020 , up from $11 \%$ in 2015 . With limited economicallyviable hydro resources left to develop, Sri Lanka is considering new coal and/or liquefied natural gas plants, both of which will require importing fuel and building associated import infrastructure.

India and Sri Lanka are separated by a narrow strait known as Palk Strait. It is 53-82 kilometers wide and connects the Bay of Bengal to Palk Bay. Connecting the two countries with a highvoltage direct current (HVDC, hereafter DC tie) transmission link has been considered for many years by both India and Sri Lanka power system planners, as well as by the broader research community (Tayal et al. 2002; Singh et al. 2013; Wijayatunga et al. 2015; Timilsin et al. 2016). For example, studies by the Asian Development Bank (Wijayatunga et al. 2015; Timilsin and Toman 2016) conclude the benefits from cross-border trade in avoided capital and operating costs and increased reliability for both countries would outweigh the project's cost. These studies, along with an analysis of trading opportunities by the South Asia Regional Initiative for Energy Integration (SARI/EI) and the Integrated Research and Action for Development (IRADe) (Singh et al. 2013), find the line could allow Sri Lanka to import power during peak periods and export excess baseload power to India during off-peak hours. 


\subsection{Study Approach}

This study aims to advance the discussion for CBET between India and Sri Lanka through a detailed simulation of coordinated system operations between the two countries, and is supported by the U.S. Department of State's Bureau of South and Central Asian Affairs Regional Connectivity Program.

Our primary tool is a production cost model, which simulates optimal unit commitment and dispatch of the system subject to physical, operational, and market constraints. There are two main benefits to modeling a full chronological time series of system operations. First, in systems with high levels of variable RE, production cost models can capture hour-to-hour variability in load and RE generation and the flexibility characteristics of each system. This will be increasingly important as wind and solar generation grows in both India and Sri Lanka. Second, production cost models can capture potential opportunities for trade and greater utilization of the most efficient generating units that arise when peak demand occurs at different times in different areas. The detailed production cost model captures the flexibility requirements necessary to balance generation and demand in every 30-minute period of the year and the cost associated with those operational decisions. This study is the first attempt to examine CBET over a full year of system operations and with greater penetration levels of RE.

This study builds on a detailed production cost model of the India power system developed under the Greening the Grid program, an initiative co-led by India's Ministry of Power and the U.S. Agency for International Development to support the iniatives taken by India's Ministry of Power for large-scale integration of RE. The model was developed by a collaborative modeling group from India's Power System Operation Corporation, Limited; National Renewable Energy Laboratory (NREL); and Lawrence Berkely National Laboratory with additional input from a wide range of experts from across the India power sector. Its initial use was for an analysis of the operational impacts of $175 \mathrm{GW}$ of variable RE on the India power system in 2022 (Palchak et al. 2017a).

Analysis of CBET required building and adding a Sri Lanka model to the India model. The Sri Lanka model was developed by NREL based on data and guidance from engineers at Ceylon Electricity Board (CEB), which is the largest electricity company in Sri Lanka and is responsible for almost all aspects of planning and operations.

\subsection{Structure of the Report}

Section 2 presents the study scenarios and methods used to create the production cost model and input databases. The results for a normal hydro year are presented in Section 3 and for dry and wet hydro weather years in Section 4. Finally, Section 5 summarizes the findings and presents final conclusions. 


\section{Study Scenarios, Modeling Methodology, and Assumptions}

The objective of this study is to evaluate the operational benefits of electrically connecting India and Sri Lanka with a DC tie that can transfer 500 megawatts (MW) of power in either direction. To meet this objective, we simulated operations of each country's power system with and without the DC tie. Additionally, to evaluate the dependency of results on Sri Lanka's hydro generation, we included a wet and dry hydro year sensitivity. The following sections describe the study scenarios, the model setup and execution, data sources and assumptions for the India and Sri Lanka power systems, and their integration into a single combined model.

\subsection{Study Scenarios}

The study considers two scenarios and three sensitivities, as outlined in Table 1. Each study scenario pairs with all three sensitivities for a total of six simulations.

Table 1. Study Scenarios and Sensitivities

\begin{tabular}{ll}
\hline Scenario & Description \\
\hline no DC & No interconnection between Sri Lanka and India \\
DC & $500 \mathrm{MW}$ interconnection between Habarana, (Sri Lanka) and Madurai (India) \\
\hline & \\
\hline Sensitivity & Description \\
\hline Dry Hydro & Hydro energy available in Sri Lanka decreased by 22\% from medium year \\
Medium Hydro & Hydro energy available as in 2014 \\
Wet Hydro & Hydro energy available in Sri Lanka increased by 22\% from medium year \\
\hline
\end{tabular}

The study scenarios are designed to evaluate the operational impacts that a DC tie would have under official projections for load growth and plans for new generation and transmission infrastructure. A major source of uncertainty for Sri Lanka's power system operations is the availability of hydropower. To understand how the operational benefits of CBET may vary under different Sri Lanka hydro conditions, the study includes three hydro sensitivities. For the dry and wet hydro sensitivities, we adjusted medium year hydro availability using monthly scalars derived from the very wet and very dry hydro scenarios developed by CEB for its Long-Term Generation Expansion Plan (CEB 2017). Table 2 provides the exact scalars.

Table 2. Monthly Scalars for Wet and Dry Hydro Years in Sri Lanka

\begin{tabular}{l|lllllllllllll}
\hline $\begin{array}{l}\text { Month } \\
\text { Wet }\end{array}$ & Jan & Feb & Mar & Apr & May & Jun & Jul & Aug & Sep & Oct & Nov & Dec & Annual \\
$\begin{array}{l}\text { Hydro } \\
\text { Dry }\end{array}$ & 1.02 & 1.18 & 1.21 & 1.19 & 1.25 & 1.23 & 1.22 & 1.26 & 1.37 & 1.27 & 1.33 & 1.08 & $\mathbf{1 . 2 2}$ \\
$\begin{array}{l}\text { Hydro } \\
\text { Hyd }\end{array}$ & 0.99 & 0.813 & 0.73 & 0.88 & 0.90 & 0.88 & 0.84 & 0.79 & 0.75 & 0.74 & 0.77 & 0.83 & $\mathbf{0 . 8 2}$ \\
\hline
\end{tabular}




\subsection{Unit Commitment and Economic Dispatch Model}

To simulate power system operations, we use a unit commitment and economic dispatch (UC\&ED) model. The model balances supply and demand every 30 minutes, consistent with the dispatch process used in Sri Lanka. By simulating UC\&ED in every 30-minute period of 2025, the study provides a full chronological picture of the year's operations. The results capture constraints that effect operator decisions, such as RE availability, generator minimum up and down times, ramp rates, and hydro max energy limits.

The UC\&ED simulation relies on the mixed integer programming software package PLEXOS Integrated Energy Model, developed by Energy Exemplar. The software commits and dispatches generators to meet load at least-cost, while adhering to thousands of physical constraints, including transmission limits, generator parameters, and reservoir limits. The India-Sri Lanka PLEXOS model has been configured to structure the mixed-integer optimization as three phases:

1. A single year-long step to plan scheduled outages.

2. A second year-long step to optimally translate multi-day hydro constraints into target end-of-day reservoir volumes.

3. 365 one-day optimization steps with a 30-minute resolution to schedule and dispatch the remaining generation to meet load. ${ }^{1}$

Although the UC\&ED simulation captures the constrained cost-minimization problem at the heart of power system operations, it relies on a few key simplifying assumptions. The model does not include load and renewable energy forecast errors and does not account for units that may be committed for reliability beyond regular ancillary service requirements. This analysis aims to assess the impacts of cross-border trade that are economically efficient and technically feasible on an already built system. As such, the UC\&ED model does not optimize investment decisions or account for fixed costs. It also assumes system operations between India and Sri Lanka are fully coordinated. Previous modeling of the India power system suggests normal between-state transaction costs of around USD 15.38/MWh (INR 1,000/MWh) (Palchak et al. 2017). We maintain this charge between India states and between India and Sri Lanka to reflect non-technical barriers to trade.

\subsection{Data Sources and Assumptions}

This study uses the India power system database developed for Greening the Grid, which simulates the operation of the India power system in 2022 with $175 \mathrm{GW}$ of RE (Palchak et al. 2017a). This database was developed and validated in collaboration with experts from across the India power sector. Details of data sources and assumptions for load, generation, and transmission are provided in Section 2 of that report.

${ }^{1}$ To emulate the decisions made in an operations room, which would weigh known changes to load or other factors beyond a single day, each one-day step solves for 48 hours and then discards the last 24 hours. 
The Sri Lanka power system database is based on data and guidance provided by CEB. Its construction followed the methodology presented in Figure 1, and is similar to what was used to build the India power system database in Greening the Grid.

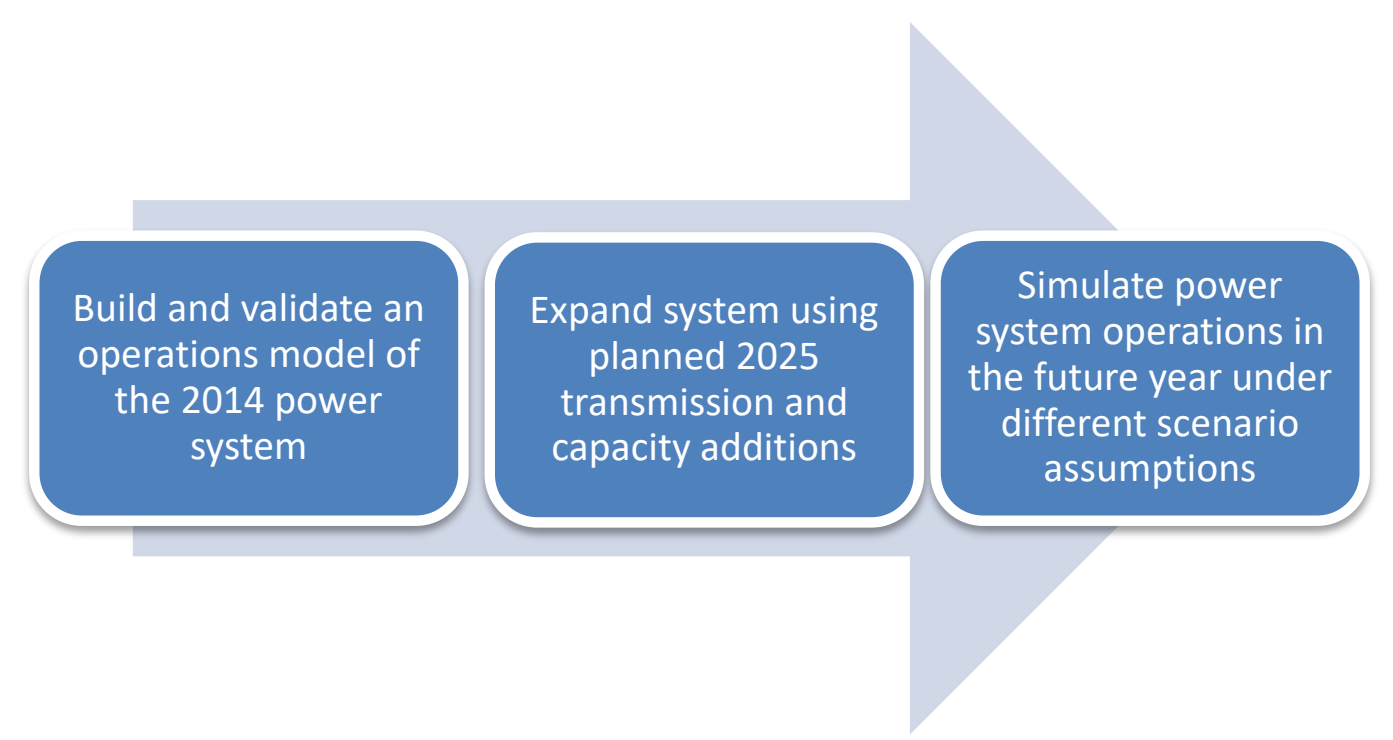

Figure 1. Process for building and simulating the 2025 Sri Lanka power system

Construction of the Sri Lanka power system database progressed in two stages: construction of a 2014 database for validation and subsequent expansion to 2025. The 2014 database is used to ensure the model inputs and constraints accurately capture the salient features of actual system operations. Using the 2014 database, we compared results from a 2014 UC\&ED simulation to historical system operations data. After an iterative process to resolve discrepancies between the modeled and historical system data, we expanded the validated 2014 database to 2025 . Figure 2 compares installed capacity by resource type between 2014 and 2025 . 


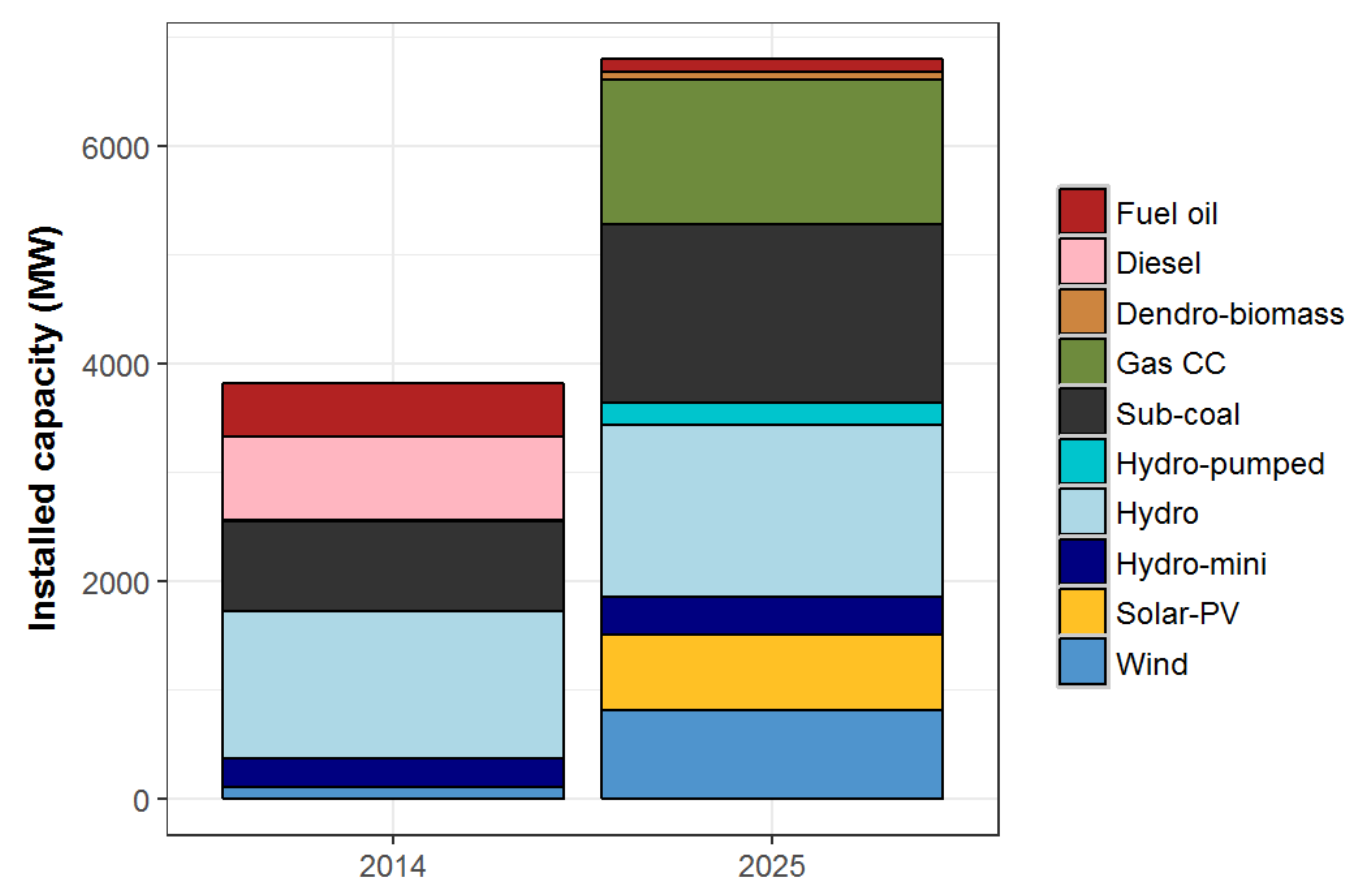

Figure 2. Comparison of installed capacity by resource type in the 2014 and 2025 Sri Lanka databases $^{2}$

Appendix A further describes the 2014 database's validation and expansion to 2025 .

\subsubsection{Thermal Generation}

Thermal generator parameters in the 2025 database are based on inputs from CEB. However, some additional assumptions were necessary to assemble the UC\&ED model inputs, particularly for independent power producer (IPP)-owned plants. Wherever possible we used plant-specific information for our database. Table 3 and Table 4 list average 2025 thermal generator parameters by category for CEB and IPP-owned generation.

${ }^{2}$ Dendro is a type of biomass used in Sri Lanka for electricity generation. 
Table 3. Average CEB 2025 Thermal Parameters ${ }^{3}$

\begin{tabular}{llll}
\hline CEB Parameter & Sub-Coal & Gas CC & Gas CT \\
\hline Total installed capacity (MW) & 1635 & 705 & 105 \\
\hline Minimum stable level (\% of capacity) & 59.9 & 48.5 & 30.0 \\
\hline Fuel cost (\$ / gigajoule [GJ]) & 2.86 & 9.48 & 9.48 \\
\hline $\begin{array}{l}\text { Heat rate-minimum stable level } \\
\text { (GJ/megawatt hour [MWh]) }\end{array}$ & 10.9 & 10.3 & 12.8 \\
\hline Heat rate-max capacity (GJ / MWh) & 9.1 & 8.3 & 12.8 \\
\hline Variable O\&M Cost (USD / MWh) & 4.5 & 12.0 & 5.5 \\
\hline Maintenance rate (\%) & 13.3 & 8.2 & 8.2 \\
\hline Forced outage rate (\%) & 7.1 & 8.1 & 8.0 \\
\hline Mean outage duration (hrs.) & & & 107 \\
Source: Western Electricity Coordinating Council 2024 & 404 & 107 & \\
Common Case & & & 1 \\
\hline Minimum up time (hrs.) & 7 & 3 & 1 \\
\hline Minimum down time (hrs.) & 5 & 3 & 14.3 \\
\hline Max ramp up (\% of capacity / minute) & 1.1 & 1.6 & 17.1 \\
\hline Max ramp down (\% of capacity / minute) & 1.1 & 1.6 & 103 \\
\hline Start cost (hot-warm-cold in USD / MW) & 257 & 127 & \\
\hline
\end{tabular}

${ }^{3}$ All averages in Table 3 and Table 4 are simple averages (not capacity weighted). Data is from CEB unless otherwise noted. 
Table 4. Average IPP 2025 Thermal Parameters

\begin{tabular}{llll}
\hline IPP Parameter & Gas CC & Gas CT & Fuel Oil \\
\hline Total installed capacity (MW) & 425 & 96 & 120 \\
\hline Minimum stable level (\% of capacity) & 41.9 & 30.0 & 37.5 \\
\hline Fuel cost (\$ / G) & 9.48 & 9.48 & 13.26 \\
\hline Heat rate - minimum stable level (GJ / MWh) & 10.3 & 12.8 & 9.3 \\
\hline Heat rate - max capacity (GJ / MWh) & 8.3 & 12.8 & 9.3 \\
\hline Variable O\&M Cost (USD / MWh) & 11.8 & 5.5 & 14.5 \\
\hline Maintenance rate (\%) & 8.2 & 8.2 & 16.4 \\
\hline Forced outage rate (\%) & 4.8 & 8.0 & 6.5 \\
\hline Mean outage duration (hrs.) & & & 107 \\
Source: Western Electricity Coordinating Council 2024 & 107 & 107 & 2 \\
\hline Common Case & & & 2 \\
\hline Minimum up time (hrs.) & 3 & 1 & 15 \\
\hline Minimum down time (hrs.) & 3 & 1 & 18 \\
\hline Max ramp up (\% of capacity / minute) & 1.6 & 20.8 & \\
\hline Max ramp down (\% of capacity / minute) & 1.6 & 20.8 & 28 \\
\hline Start cost (hot-warm-cold in USD / MW) & 127 & 150 & \\
\hline
\end{tabular}

Combined cycle gas generators are modeled as coupled two-part units such that heat output from the combustion turbine (reduced by a boiler efficiency) serves as heat input for the corresponding steam turbine.

Fuel costs are those used for CEB's 2018 Long-Term Generation Expansion Plan and are assumed to be the same for IPP and CEB-owned plants. Cost differences between generators of the same fuel type are due to variation in heat rates and variable operation and maintenance costs. Figure 3 presents a merit order for Sri Lanka's 2025 thermal generators when operating at max capacity (all costs in USD / MWh). 


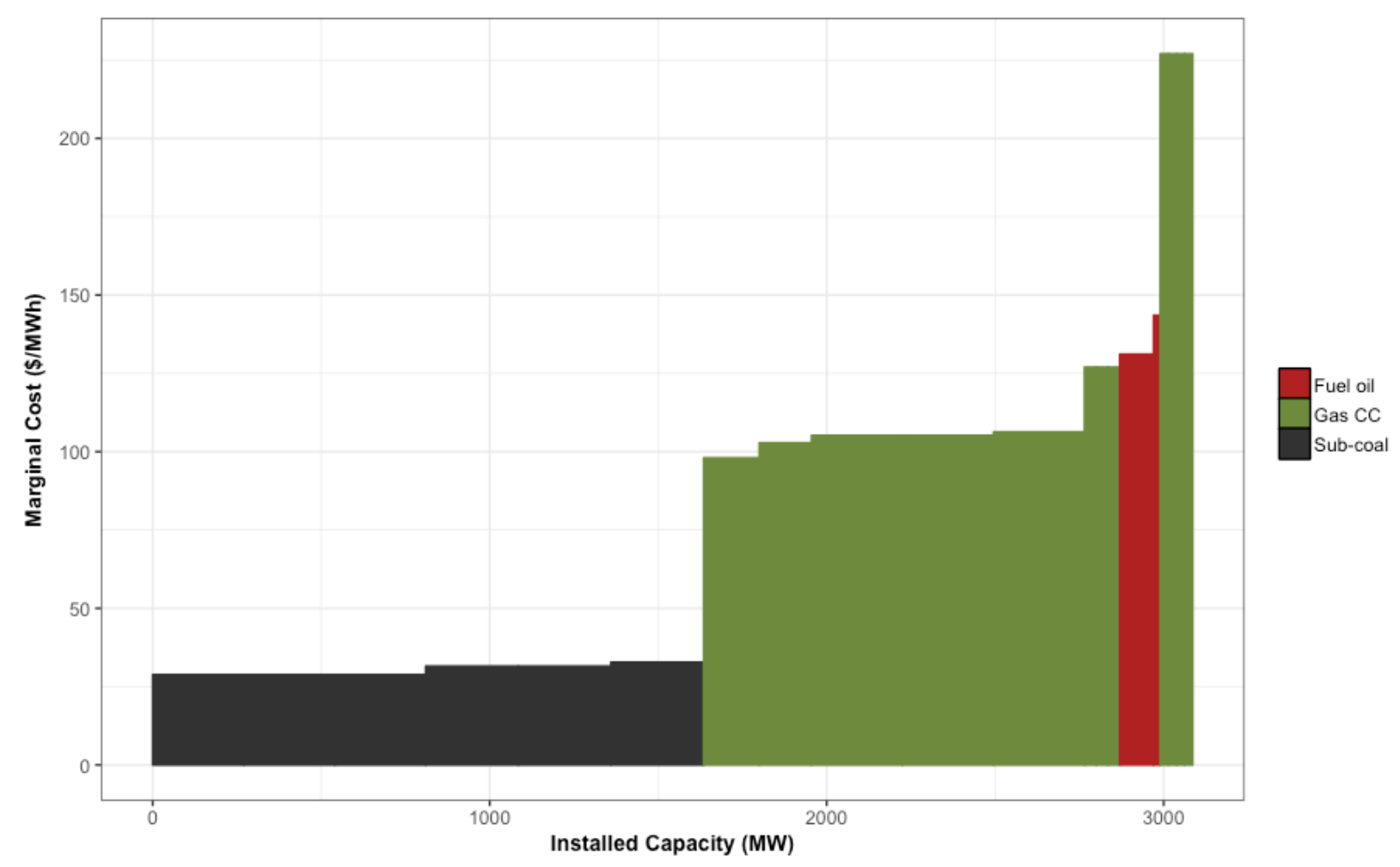

Figure 3. 2025 merit order for Sri Lanka's thermal generators operating at max capacity

\subsubsection{Hydro Generation}

Representing hydro generation in a UC\&ED model is inherently difficult for a variety of reasons, including:

- Hydro plant operations must consider multiple time scales of minutes and hours and how much water should be stored for future use over several weeks and months.

- Available capacity is subject to seasonal and inter-annual changes in rainfall and inflows as well as losses due to evaporation, silting, and seepage.

- Water use for electricity generation is constrained by competing water demand for other uses such as irrigation and recreation.

- Operational planning for plants on the same river must consider interdependencies between upstream and downstream plants.

The 2025 Sri Lanka database contains a detailed representation of Sri Lanka's hydroelectric system based on 2014 operations. Treating 2014 as a medium hydro year, the 2025 wet and dry hydro sensitivities described in section 4 simulate the effect on operations if hydro availability were to deviate significantly from 2014 levels.

Most of the major hydro generators in Sri Lanka are located along the Kelani and Mahaweli river basins (Figure 4 and Figure 5). Table 5 summarizes large 2025 Sri Lanka hydro generator parameters. In the model, each plant receives water into its head storage from the generator immediately upstream (along with additional inflows from intermediate tributaries), which it in turn releases downstream to produce electricity. A hydro plant's storage size determines the temporal scale of its flexibility. Plants with multi-day storage capacity use the initial year-long 
UC\&ED simulation phase described in section 2.2 to plan target generation and reservoir levels, allowing them to consider future water value during the 365 single-day optimization steps. Runof-river or small pondage plants have little to no flexibility and must release water at roughly the rate of the dam directly upstream, with a one-hour waterway traversal time delay.

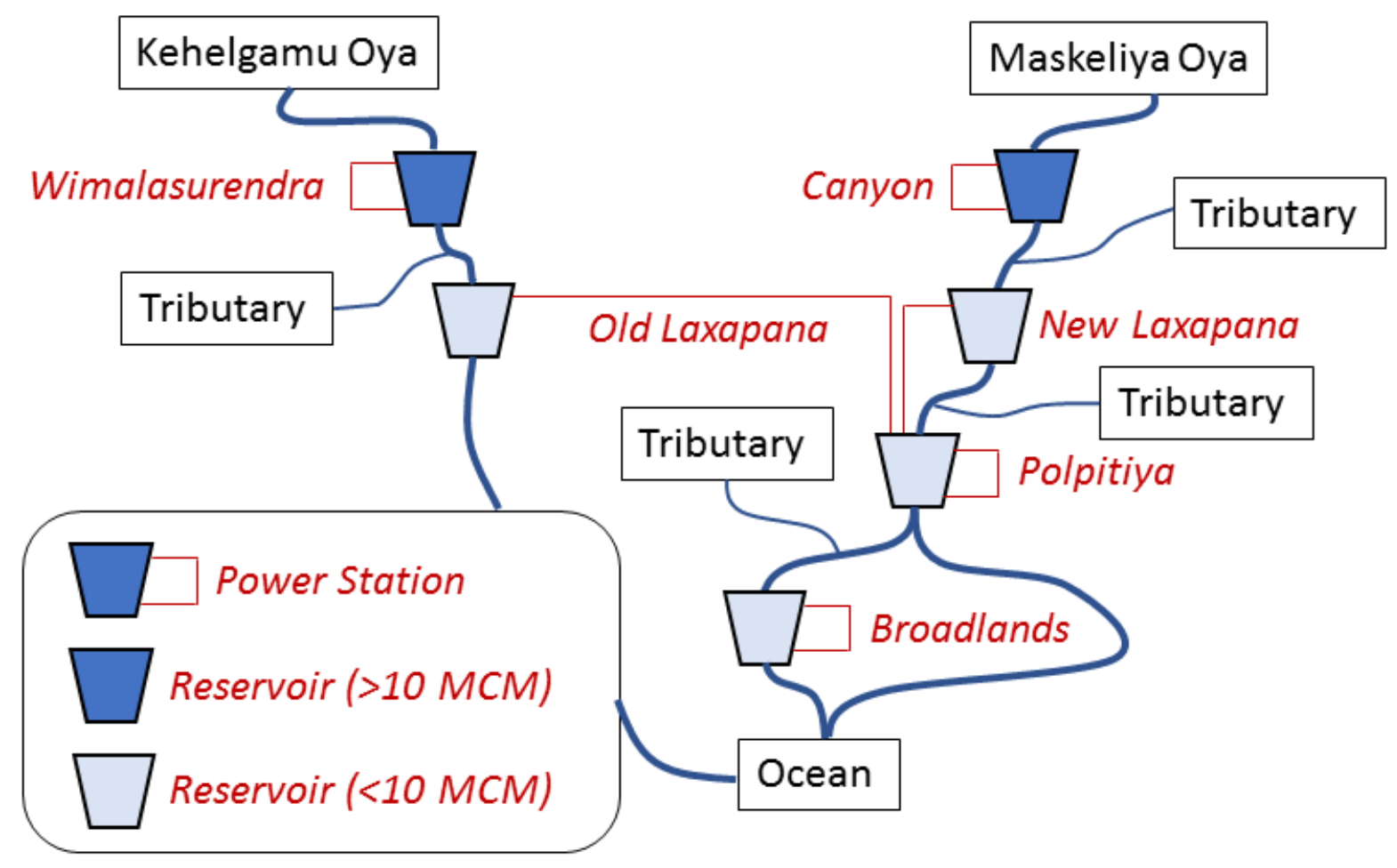

Figure 4. Hydropower system in Kelani River Basin 


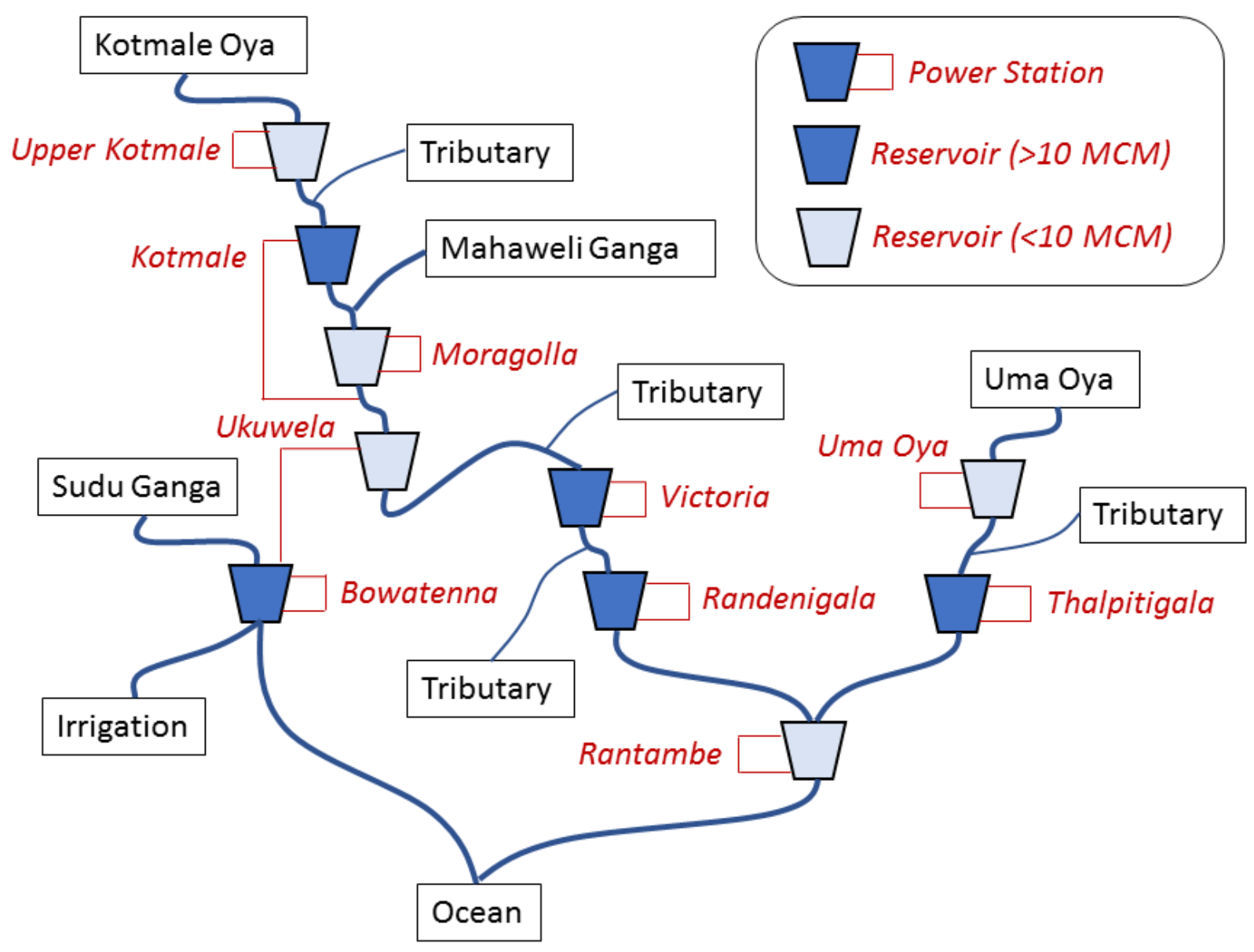

Figure 5. Hydropower system in Mahaweli River Basin 
Table 5. Sri Lanka Hydro Generator Parameters and Operating Constraints

\begin{tabular}{|c|c|c|c|c|c|c|}
\hline Generator & $\begin{array}{l}\text { Capacity } \\
\text { (MW) }\end{array}$ & $\begin{array}{l}\text { Minimum } \\
\text { Stable Level } \\
(\%)\end{array}$ & $\begin{array}{l}\text { Storage } \\
\text { Volume } \\
\text { (MCM) }\end{array}$ & $\begin{array}{l}\text { Efficiency } \\
\text { (MW / m3/s) }\end{array}$ & $\begin{array}{l}\text { Outage Rate } \\
(\%)\end{array}$ & $\begin{array}{l}\text { Operating } \\
\text { Constraints }\end{array}$ \\
\hline Wimalasurendra & $2 \times 25$ & 19 & 49.1 & 1.90 & 5 & \\
\hline Canyon & $2 \times 25$ & 22 & 127.1 & 1.68 & 5 & \\
\hline New Laxapana & $2 \times 50$ & 10 & 0.6 & 3.84 & 10 & $\begin{array}{l}5 \mathrm{MW} \\
\text { minimum load } \\
\text { requirement }\end{array}$ \\
\hline Old Laxapana & 53.5 & 7 & 0.2 & 4.40 & 5 & $\begin{array}{l}5 \mathrm{MW} \\
\text { minimum load } \\
\text { requirement }\end{array}$ \\
\hline Polpitiya & $2 \times 37.5$ & 7 & 0.1 & 2.27 & 10 & $\begin{array}{l}5 \mathrm{MW} \\
\text { minimum load } \\
\text { requirement }\end{array}$ \\
\hline Upper Kotmale & $2 \times 75$ & 29 & 0.8 & 4.41 & 5 & \\
\hline Kotmale & $3 \times 67$ & 16 & 175 & 1.85 & 5 & \\
\hline Ukuwela & $2 \times 20$ & 50 & 4.1 & 0.70 & 1 & \\
\hline Victoria & $3 \times 70$ & 10 & 721 & 1.66 & 5 & \\
\hline Bowatenna & 40 & 25 & 52 & 0.43 & 1 & \\
\hline Rantambe & $2 \times 25$ & 30 & 4.4 & 0.28 & 1 & \\
\hline Randenigala & $2 \times 60$ & 37 & 863 & 0.66 & 5 & \\
\hline Samanalawewa & $2 \times 60$ & 8 & 228 & 2.70 & 5 & \\
\hline Kukule & $2 \times 37.5$ & 38 & 1.7 & 1.72 & 5 & \\
\hline Inginiyagala & 11.25 & & & & 5 & \\
\hline Broadlands & 35 & 56 & 0.2 & 0.50 & 1 & \\
\hline Gin Ganga & 20 & & & & 1 & \\
\hline Moragolla & 27 & 83 & 2 & 0.66 & 1 & \\
\hline Seethawaka & 20 & & & & 1 & \\
\hline Thalpitigala & 15 & 91 & 11.4 & 0.88 & 1 & $\begin{array}{l}60 \% \text { inflows } \\
\text { diverted for } \\
\text { irrigation }\end{array}$ \\
\hline Uma Oya & $2 \times 60$ & 100 & 0.7 & 6.22 & 1 & \\
\hline
\end{tabular}

(Source: Data provided by CEB )

Some plants required special irrigation or miscellaneous constraints as summarized in Table 5. All hydro plants also have a minimum down time of 6 hours to limit rapid cycling and a mean outage duration of 1 week.

To simulate generation constraints due to water availability at different times of the year, each large hydro plant has a natural inflow profile derived from 2014 CEB data. Natural inflow 
represents the amount of water reaching a hydro plant's head storage above and beyond that which it receives from the generator directly upstream. Initial testing showed that constraining hydro generation only by natural inflows caused modeled 2014 hydro generation to exceed historical levels by $34 \%$, suggesting that water losses through evaporation or waterway diversion may be preventing all water flowing into a cascade from reaching the sea. To more accurately calibrate hydro generation with inflows, actual 2014 generation levels were used to create monthly maximum energy limits on large hydro plants.

In addition to the 1,583 MW of large CEB-owned hydro, we include $352 \mathrm{MW}$ of independentlyowned mini-hydro plants expected to exist in 2025. Under current rules, mini-hydro generation is a must-take resource. Generation from mini-hydro plants is fixed based on monthly average capacity factors presented in CEB's Long-Term Generation Expansion Plan. Total contributions from mini-hydro generation are estimated to be 902 gigawatt hours (GWh) in 2014 and 1,369 GWh in 2025.

The 2025 database also includes a 200 MW pumped storage plant planned on the Maha Oya river. ${ }^{4}$ Based on design parameters from $\mathrm{CEB}$, the plant is modeled with a pump efficiency of $70 \%$ and mean turbine efficiency of $4.21 \mathrm{MW} / \mathrm{m}^{3} / \mathrm{sec}$. The head and tail storage, 3.15 and 3.2 cubic hectometers, respectively, are sufficient for the pumped storage plant to generate at max capacity for 6 hours.

\subsubsection{Transmission Network}

Information on the transmission network in the 2025 Sri Lanka PLEXOS model comes from a set of 2025 Power System Simulator for Engineering (PSS/E) files supplied by CEB. Table 6 summarizes the number of transmission lines and nodes by voltage (kilovolt) included in the 2025 model. Power flows are calculated using a linearized DC optimal power flow algorithm.

Table 6. Basic Information on Modeled 2025 Sri Lanka Transmission Network

\begin{tabular}{lll}
\hline Voltage & \# of Lines & \# of Nodes \\
\hline 220 kilovolts & 109 & 58 \\
132 kilovolts & 269 & 186 \\
$<132$ kilovolts & - & 156 \\
\hline
\end{tabular}

The UC\&ED model also assumes that India experiences no intrastate transmission congestion. Elimination of intrastate transmission constraints in India and subsequent reduction of the India network into 36 state-wide nodes connected by interstate transmission greatly reduces the model's computation time. The Sri Lanka model is run with internal transmission intact, to allow an analysis of whether internal transmission is sufficient to allow for full use of the $500 \mathrm{MW}$ DC tie capacity.

${ }^{4}$ CEB plans to build $600 \mathrm{MW}$ of pumped storage capacity on the Maha Oya, however only the first $200 \mathrm{MW}$ phase is projected to be finished by 2025 . 


\subsubsection{Wind and Solar Sites}

In 2014, Sri Lanka had an installed wind capacity of $126 \mathrm{MW}$ and solar capacity of $1 \mathrm{MW}$, whose locations in the 2014 model come from the PSS/E files and CEB's Long-Term Generation Expansion Plan. To identify planned additions to wind and solar capacity between 2014 and 2025, we used information in the PSS/E files and CEB reports. Table 7 lists the wind and solar expansion details in the model. Each 'site' represents a distinctly modeled RE installation with its own generation profile. Wind and solar capacities and coordinate locations from CEB serve as the basis for creating wind and solar generation profiles, as described in section 2.3.5.

Table 7. Sri Lanka Database Wind and Solar Capacity

\begin{tabular}{llll}
\hline Year & Wind (Sites / MW) & Utility-Scale PV (Sites / MW) & Rooftop PV (Sites / MW) \\
\hline 2014 & $7 / 126$ & $1 / 1.2$ & $0 / 0$ \\
\hline 2025 & $37 / 813$ & $43 / 491$ & 64 / 200 \\
\hline
\end{tabular}

The UC\&ED model treats rooftop-PV as a must-take resource like mini-hydro, while utilityscale PV and wind are curtailable. ${ }^{5}$ CEB's Long-Term Generation Expansion Plan includes a target of $200 \mathrm{MW}$ of rooftop PV by 2025.

\subsubsection{Load, Wind, and Solar data}

Instantaneous weather conditions impact both electricity demand and variable renewable generation. To capture this correlation, it is important to use load, wind, and solar data based on the same weather year in the UC\&ED model. Rather than attempt to anticipate future weather conditions, the model assumes that 2014 conditions will prevail in 2025. The approach of assuming past weather in a future year to synchronize wind, solar, and load data is a wellestablished procedure in power system modeling (Milligan et al. 2012).

Solar generation profiles used in the model originate from 2014 National Solar Radiation Database data. The database consists of one-hour 10 by 10-kilometer resolution solar irradiance measurements derived from satellite observations, which we linearly interpolated to half-hourly resolution to match Sri Lanka's dispatch timeframe. We utilized NREL's System Advisor Model (SAM) to convert solar irradiance into site-specific solar generation profiles using physical assumptions about current and future solar installations summarized in Table 8.

\footnotetext{
${ }^{5}$ Curtailment represents available energy from wind or PV resources that cannot be used to serve load, due to constraints such as inflexible generation or insufficient transmission. This report uses the term "curtailment" only for wind and solar, and not for load.
} 
Table 8. Solar PV Assumptions in SAM

\begin{tabular}{lll}
\hline Parameter & Utility-PV & Rooftop-PV \\
\hline DC to AC ratio & 1.1 & 1.2 \\
Fixed-tilt & Latitude & $5^{\circ}$ \\
Azimuth & $180^{\circ}$ & $180^{\circ}$ \\
Inverter efficiency & $96 \%$ & $96 \%$ \\
Losses & $14 \%$ & $14 \%$ \\
Ground cover ratio & 0.4 & 0.4 \\
\hline
\end{tabular}

Wind generation profiles originate from 2014 outputs of the Weather Research and Forecasting model, which produces weather data at five-minute intervals with a geographic resolution of 3 by 3 kilometers. From this data set, we extracted wind speeds at 50-, 80-, 100-, and 120-meter hub heights, corresponding to the heights of existing and proposed wind sites in Sri Lanka, and used SAM to convert wind speed and other Weather Research and Forecasting model parameters to power generation. In SAM, we assumed 17\% losses and generic power curves for several turbine manufacturers. We calculated a rolling 30-minute average to aggregate from 5-minute to halfhourly resolution.

The 2014 Sri Lanka power system database includes actual half-hourly 2014 demand. CEB also provided forecast half-hourly 2025 demand. To retain the relationship between 2014 weather patterns and demand, while also capturing anticipated load growth, we created a new 2025 load forecast using 2014 as the base year. The 2014 load was increased using half-hourly scaling factors determined on a weekly basis based on the change in the demand for an average day per week between 2014 and 2025. Applying the weekly scalars results in a 2025 load profile with a peak demand of 4,005 MW and total demand of 23,480 GWh while maintaining correlations with 2014 load, wind, and solar data. Each modeled power system bus receives a portion of the overall demand profile based in its load participation factor, a scalar designed such that the sum of load participation factors across all nodes equals one. Load participation factors for Sri Lanka in 2025 come directly from CEB.

Figure 6 compares Sri Lanka's average hourly available wind, solar, and mini-hydro energy in 2025 to its average hourly load. 


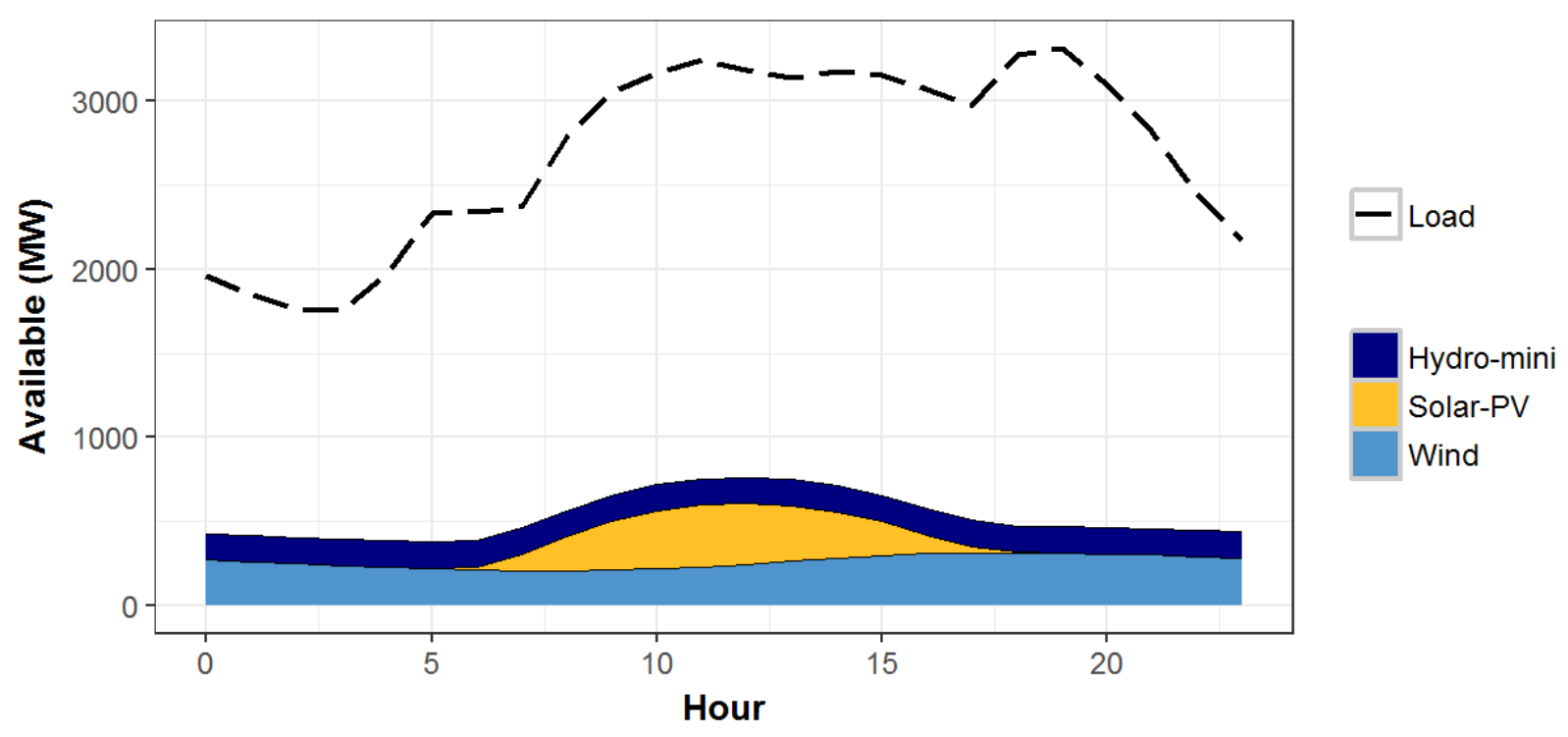

Figure 6. Comparison of Sri Lanka's average hourly available wind, solar, and mini-hydro energy and load in 2025

\subsubsection{Reliability Constraints}

The Sri Lanka UC\&ED model holds an upwards reserve requirement equal to $5 \%$ of system load. PLEXOS does not dispatch reserves, but simply ensures that enough headroom exists in the online capacity. Also integrated into the model is Sri Lanka's operational rule that no single generator can supply more than $25 \%$ of instantaneous demand. This rule applies to flows across the DC tie as well as Sri Lanka's domestic generating units.

\subsection{Integrating the India and Sri Lanka Models}

The independently developed India and Sri Lanka power system databases are not inherently compatible. We apply currency, volume, and penalty function conversions according to Table 9 such that the combined 'South Asia' model functions according to a uniform set of rules. The currency conversion is INR $65=\mathrm{USD}$, a rough approximation of the exchange rate over recent years but accurate enough to preserve the relative merit order between the two countries. 
Table 9. Conversions Applied When Integrating the India and Sri Lanka Models into a Combined 'South Asia' Model

\begin{tabular}{llll}
\hline Unit & India & Sri Lanka & Combined South Asia \\
\hline Currency & INR & USD & USD \\
Volume unit & GWh & Cumec day & Cumec day \\
Value of lost load & INR $1 \mathrm{crore}^{7}$ & USD 1M & USD 1M \\
Value of reserve shortage & INR 0.4 crore & USD 230 & USD 400,000 \\
Hydro minimum load penalty & INR 500,000 & USD 50,000 & USD 50,000 \\
\hline
\end{tabular}

The above conversions ensure that the two databases are mechanically compatible. However, the usefulness of results from a joint UC\&ED simulation depends on three further considerations:

1. Wind, solar and load data compatibility. Wind and solar generation profiles for both India and Sri Lanka are sourced from the same 2014 Weather Research and Forecasting model and National Solar Radiation Database data. They correlate with the 2014 load data provided by CEB and India's Central Electricity Authority.

2. Generator costs. India database generator costs are based on 2014-2016 variable cost data collected from India's Regional and State Load Dispatch Centers. Sri Lanka database fuel costs are those used for CEB's 2018 Long-Term Generation Expansion Plan. From a roughly equivalent cost basis, the study assumes that inflation, fuel price changes, and fluctuations in exchange rates with the USD affect both countries equally.

3. Generation and transmission expansion. While the Sri Lanka database uses 2025 expansion plans from CEB, the existing India database uses 2022 expansion plans from the Central Electricity Authority. Expanding the India database to 2025 to match Sri Lanka is beyond the study's scope. However, a DC interconnection study of the two systems remains valid because of the common basis in cost, load, and variable generation. The key assumption made by not expanding India to 2025 is that India's generation mix relative to load changes little between 2022 and 2025.

4. Transaction charge - Transaction charges, or hurdle rates, imposed on interregional power flows are a modeling technique used to capture non-physical effects or transaction costs that may limit transmission utilization, such as differences in scheduling practices. Previous modeling of the Indian power system suggests normal interstate transaction costs of around INR 1000/MWh (Palchak et al. 2017). We maintain this hurdle rate between Indian states. We also apply it to power flows across the DC tie between Sri Lanka and India.

\footnotetext{
${ }^{6}$ A cumec day refers to the volume of water that would accumulate if one cubic meter per second (cumec) of water were to flow for a day.

${ }^{7}$ Crore is a term used commonly on the India subcontinent to denote 10 million $\left(10^{7}\right)$.
} 


\section{Results}

In the following section, we analyze the impact of CBET via a $500 \mathrm{MW}$ DC tie on India and Sri Lanka's 2025 electricity system operations under medium hydro conditions. We place emphasis on the following metrics:

1. Electricity production cost

2. Electricity generation by resource

3. Thermal fleet operations

4. Hydro fleet operations

5. Implications for wind and solar integration

6. DC tie utilization

7. Internal transmission flows

8. Example system operations.

Hereafter, table and figure legends reference the production cost simulation without a DC tie as 'no DC' and the production cost simulation with a DC intertie as 'DC'.

\subsection{Electricity Production Cost}

The addition of a DC tie between India and Sri Lanka results in an overall system savings of $0.5 \%$, or roughly USD 180 million annually. This result is a combination of a sharp decline in Sri Lanka's production cost offset by a slight rise in India's. The slight rise in India's total production cost is due to an increase in total generation in India. This will be discussed more in the next section. Table 10 compares the total and per-unit production cost (in millions of USD and USD/MWh) of operating Sri Lanka and India's electricity systems in 2025 with and without a DC tie. ${ }^{8}$

India and Sri Lanka's combined electricity production costs fall by $0.5 \%$ or USD 180 million with CBET.

\footnotetext{
${ }^{8}$ The study does not address the question of how to allocate the combined 180 million in savings between India and Sri Lanka, but rather demonstrates the potential savings available across both countries if a DC tie were built.
} 
Table 10. Total (\$M) and Per-Unit (\$/MWh) Electricity Production Cost, with and without DC Tie, India and Sri Lanka

\begin{tabular}{llll}
\hline Total Production Cost & No DC $\mathbf{( \$ M )}$ & DC $(\mathbf{\$ M})$ & Change in Cost (\%) \\
\hline India & 34,430 & 34,480 & 0.2 \\
Sri Lanka & 690 & 450 & -34.6 \\
\hline Total & 35,120 & 34,940 & -0.5 \\
\hline
\end{tabular}

\begin{tabular}{|c|c|c|c|}
\hline Per-Unit Production Cost & No DC (\$/MWh) & DC (\$/MWh) & Change in Cost (\%) \\
\hline India & 20.9 & 20.9 & 0.0 \\
\hline Sri Lanka & 29.0 & 21.3 & -26.8 \\
\hline Total & 21.0 & 20.9 & -0.5 \\
\hline
\end{tabular}

Roughly $98.7 \%$ of operational costs in the combined India and Sri Lanka system arise from fuel and other variable operation and maintenance costs. The remaining $1.3 \%$ are start costs, which are costs incurred from starting a thermal unit. While combined fuel and other variable costs fall by $0.5 \%$ with the DC tie, start costs fall by $3.0 \%$. The proportionally higher decline in start costs implies reduced stress on the thermal fleet, an effect that will be further examined in Section 3.3.

\subsection{Electricity Generation by Resource}

\subsection{TWh of imports from India cause natural gas, dendro biomass, and fuel oil generation in Sri Lanka to fall by $69 \%, 74 \%$ and $92 \%$, respectively.}

With a DC tie, imported electricity from India displaces more expensive thermal generation in Sri Lanka, driving the above-mentioned 34.6\% reduction in its production cost. Figure 7 compares annual electricity generation and plant load factor (PLF) by resource type in Sri Lanka with and without a DC tie. While coal generation increases slightly $(0.9 \%)$ with a DC tie, relatively more expensive natural gas, dendro biomass, and fuel oil generation fall by $69.2 \%$, $74.3 \%$, and $92.1 \%$, respectively. Their replacement with 2.9 terawatt-hours (TWh) of imports accounts for the majority of combined cost savings. ${ }^{9}$ Imports displace gas rather than coal because, as shown in Figure 3, gas generators in Sri Lanka have a higher variable operating cost than coal.

\footnotetext{
${ }^{9}$ Sri Lanka also exports 0.3 TWh to India as discussed in section 3.6, resulting in total annual net imports of 2.6 TWh.
} 

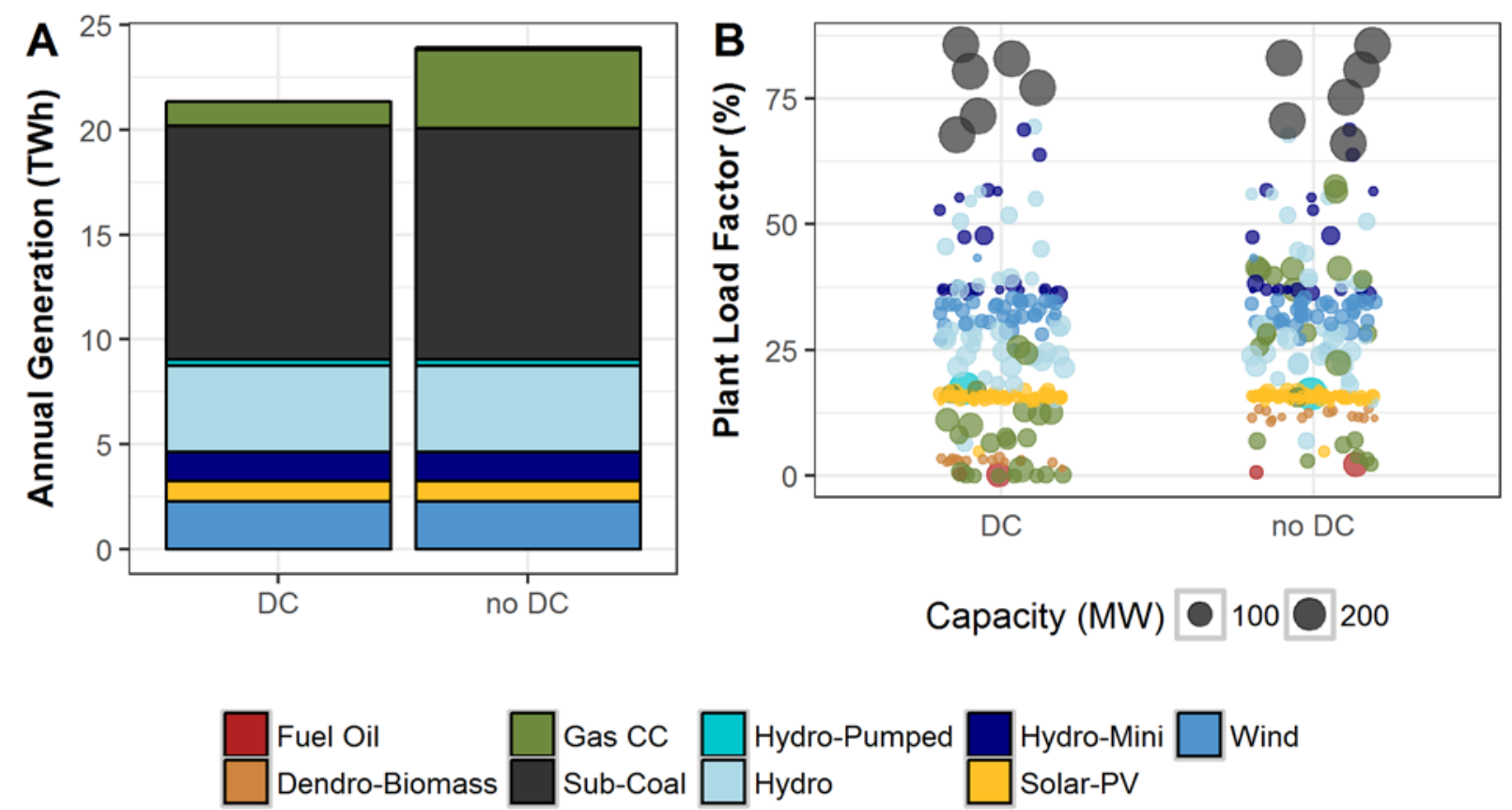

Figure 7. Generation and plant load factor by resource type in Sri Lanka, with and without a DC tie.

Note: Dots on the right-hand plot represent individual plants sized to nameplate capacity and colored by fuel type.

To export 2.9 TWh to Sri Lanka, India increases coal generation in the Western, Southern, and Eastern regions and RE generation (through reduced curtailment) in the Southern region.

To provide 2.9 TWh of exports to Sri Lanka across the DC tie, India increases its generation from coal $(2.0 \mathrm{TWh})$, wind and solar $(0.4 \mathrm{TWh})$, and natural gas $(0.2 \mathrm{TWh})$. Rather than increase coal generation only from Southern region plants close to the connection point with Sri Lanka, the additional energy comes from multiple states, particularly Chhattisgarh (700 GWh) and Odisha ( $400 \mathrm{GWh})$. The location-dependence reflects broad variations in the cost of India coal generation.

Because of constraints related to thermal plant flexibility, limited interconnections with other regions, and high RE penetrations relative to load, India's Southern region is more susceptible to RE curtailment than other regions (Palchak et al. 2017b). With no DC tie, the Southern region accounts for $97.7 \%$ (5.1 TWh) of India's overall RE curtailment. CBET with Sri Lanka provides an additional export avenue for the Southern region's surplus RE generation.

Figure 8 summarizes how generation by resource type changes in the four main India power grid regions and Sri Lanka with the addition of a DC tie. 


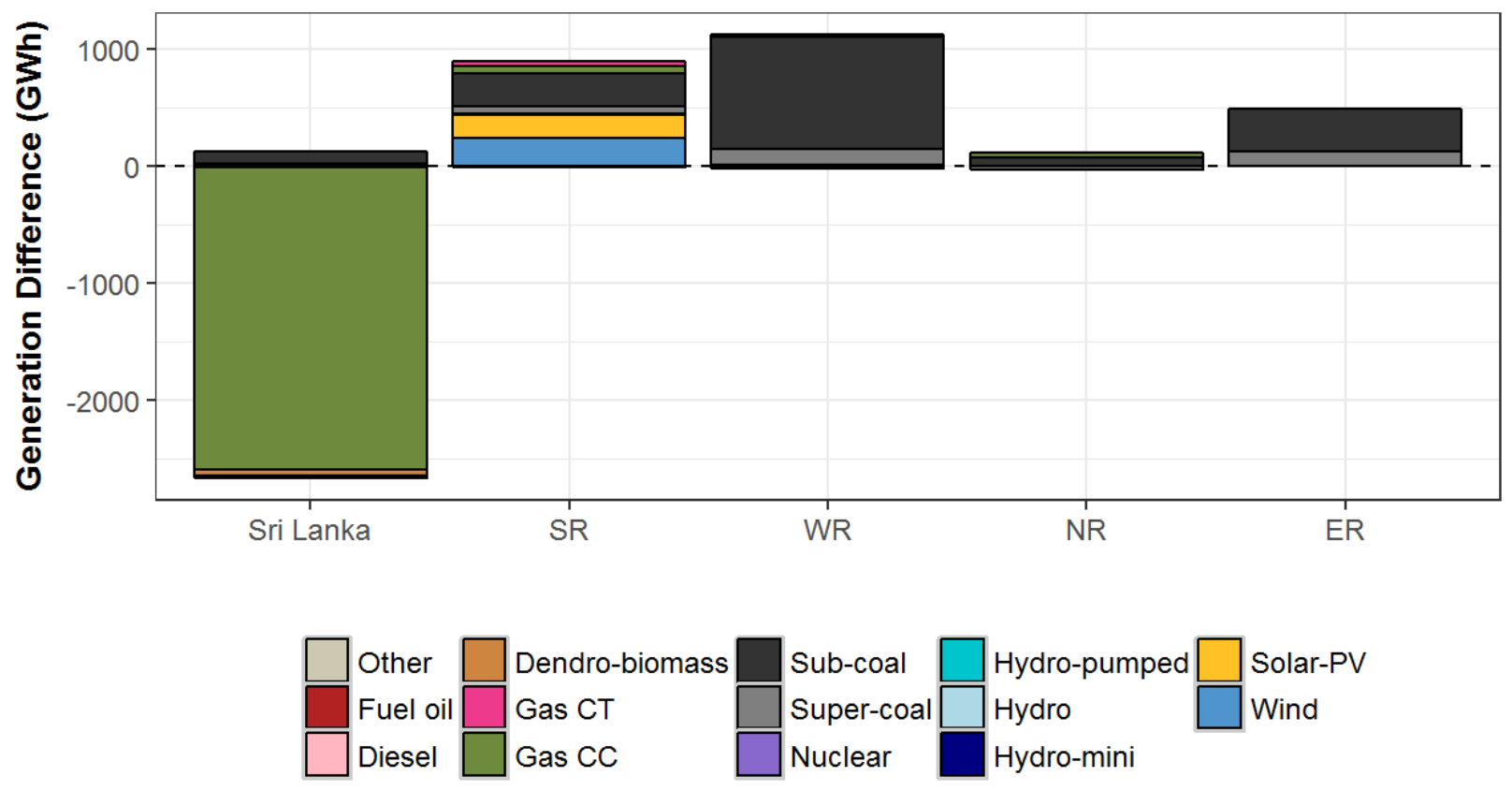

Figure 8. Change in generation by resource type with a DC tie, 4 India regions and Sri Lanka.

(India regions: SR - Southern region; WR - Western region; ER - Eastern region; NR - Northern region.)

\section{Production cost savings from the DC tie occur disproportionally during Sri Lanka's low} hydro months.

Benefits from the DC tie occur disproportionally from reducing Sri Lanka's thermal generation during the low hydro months. The three panels in Figure 9 compare (A) Sri Lanka's hydro availability, (B) Sri Lanka's change in generation with a DC tie, and (C) overall production cost savings by month. The three lowest hydro months (February, March, and April) account for $34.6 \%$ of Sri Lanka's overall production cost savings with the DC tie. In October, additional imports across the DC tie offset higher-than-average coal outages in Sri Lanka. In December, high hydro availability in Sri Lanka creates cost-effective export opportunities from Sri Lanka to India. 

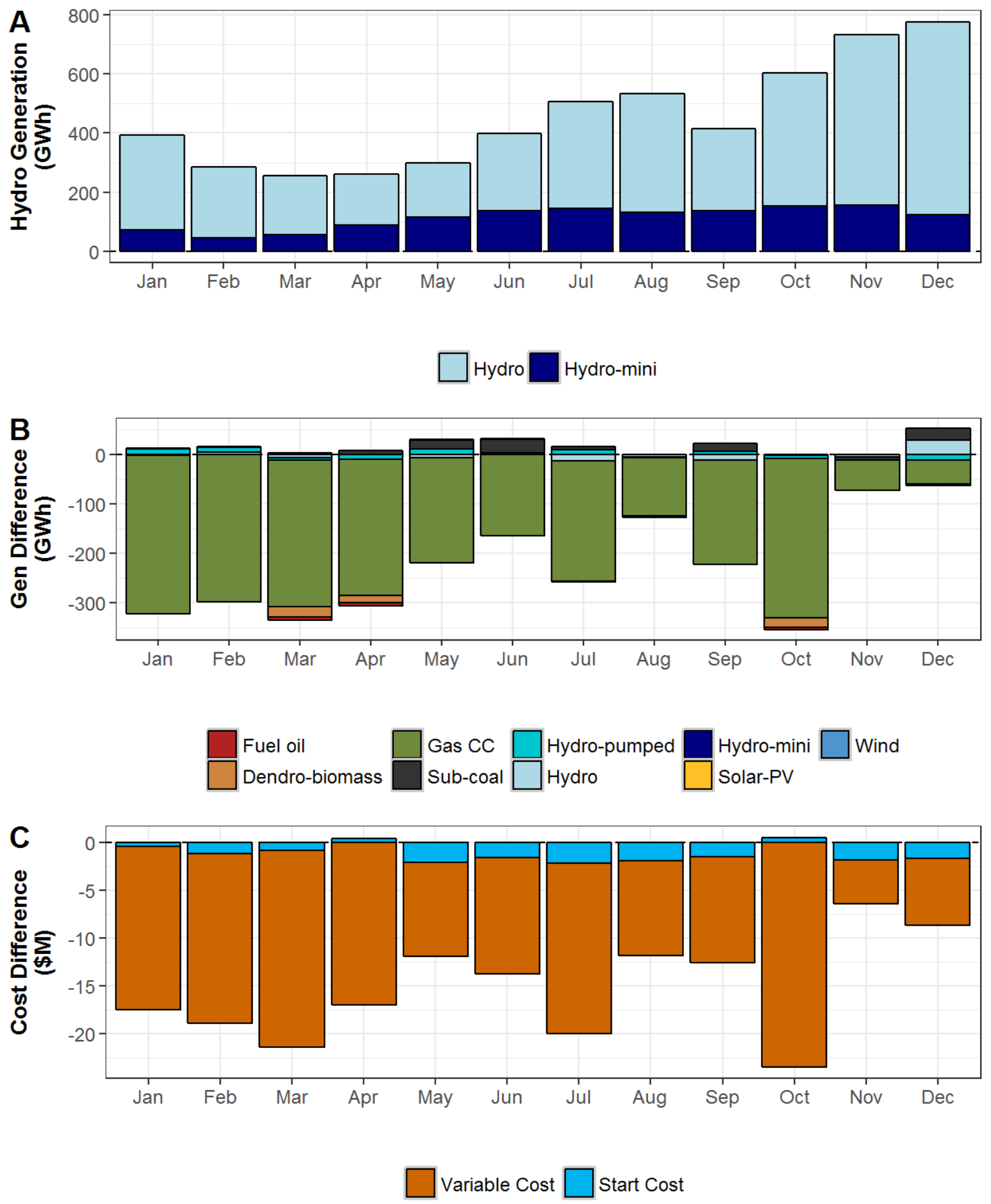

Figure 9. Comparison by month of (A) Sri Lanka's hydro availability, (B) Sri Lanka's change in generation with a DC tie, and (C) production cost savings across both Sri Lanka and India with a DC tie 


\subsection{Thermal Fleet Operations}

CBET causes India and Sri Lanka's combined generator start costs to fall by 3.0\%, suggesting that the DC tie can reduce the cycling and ramping stress placed on the countries' combined thermal fleets. Frequent ramping, operation at minimum stable level, and cycling can increase operating costs and accelerate the depreciation of thermal generators designed for less variable operation (Kumar et al. 2012). Especially in India's Southern region, where maximum instantaneous RE penetrations could exceed $85 \%$ of load in 2022 (Palchak et al. 2017b), CBET could reduce the flexibility demands of some of India's thermal generators. Table 11 and Table 12 summarize the DC tie's effect on thermal fleet operations in India and Sri Lanka, including starts (the starting of an offline unit), time spent at minimum generation, and average days on per start.

Because of the relative sizes of the two systems, improvements to aggregate thermal fleet operations in India from an interconnection with Sri Lanka are minor (impacts to individual units were not evaluated). In Sri Lanka, coal generators experience slightly fewer starts and spend less time at the minimum stable level while generating $0.9 \%$ more energy, resulting in a $0.2 \%$ reduction in the average cost per megawatt hour (MWh) of coal generation. However, the average per-MWh production cost of non-coal thermal generators in Sri Lanka increases 3.8\% with a DC tie, because non-coal thermal generators in Sri Lanka see a steep decline in PLF relative to a more modest decline in starts. With a DC tie, previously middle-merit-order gas generators operate as peaking plants, with shorter on-cycles and more time offline.

Table 11. Summary of Coal Fleet Operations in India and Sri Lanka without and with DC Tie (No DC / DC), Capacity Weighted Averages

\begin{tabular}{lllll}
\hline Coal & PLF (\%) & $\begin{array}{l}\text { Starts } \\
\text { (\#) Per } \\
\text { Unit }\end{array}$ & $\begin{array}{l}\text { Minimum Stable Level } \\
\text { (\% of Year) }\end{array}$ & $\begin{array}{l}\text { Avg. Time on Per Start } \\
\text { (Days) }\end{array}$ \\
\hline Sri Lanka & $76.7 / 77.4$ & $5.2 / 5.0$ & $3.2 / 1.2$ & $65.7 / 65.5$ \\
Tamil Nadu & $45.1 / 45.1$ & $7.8 / 7.7$ & $18.7 / 18.4$ & $38.3 / 38.7$ \\
Southern region & $43.5 / 43.5$ & $7.8 / 7.6$ & $19.0 / 18.8$ & $47.0 / 47.3$ \\
India & $50.2 / 50.3$ & $6.3 / 6.3$ & $12.2 / 12.1$ & $54.1 / 54.4$ \\
$\begin{array}{l}\text { Combined South } \\
\text { Asia }\end{array}$ & $50.4 / 50.5$ & $6.3 / 6.3$ & $12.1 / 12.1$ & $54.2 / 54.5$ \\
\hline
\end{tabular}


Table 12. Summary of Other Thermal ${ }^{10}$ Operations in India and Sri Lanka with and without DC Tie (No DC / DC), Capacity Weighted

\begin{tabular}{lllll}
\hline Other Thermal & PLF (\%) & $\begin{array}{l}\text { Starts }(\#) \\
\text { Per Unit }\end{array}$ & $\begin{array}{l}\text { Minimum Stable } \\
\text { Level (\% of Year) }\end{array}$ & $\begin{array}{l}\text { Average Time on Per } \\
\text { Start (Days) }\end{array}$ \\
\hline Sri Lanka & $28.7 / 8.8$ & $83.2 / 48.4$ & $6.5 / 3.4$ & $1.7 / 0.8$ \\
\hline Tamil Nadu & $26.0 / 26.2$ & $77.0 / 72.4$ & $8.9 / 8.8$ & $1.2 / 1.3$ \\
\hline Southern region & $12.5 / 12.6$ & $53.7 / 50.1$ & $5.1 / 5.1$ & $0.7 / 0.8$ \\
\hline India & $14.1 / 14.2$ & $46.4 / 45.1$ & $15.6 / 15.6$ & $5.9 / 5.8$ \\
\hline $\begin{array}{l}\text { Combined South } \\
\text { Asia }\end{array}$ & $14.9 / 13.9$ & $48.4 / 45.3$ & $15.1 / 15.0$ & $5.6 / 5.5$ \\
\hline
\end{tabular}

Figure 10 and Figure 11 show coal and other thermal fleet operations in Sri Lanka with and without the DC tie. Figure 10 shows the modest improvements to coal operations while Figure 11 illustrates the steep decline in gas and other thermal PLFs against a more modest decline in generator starts.
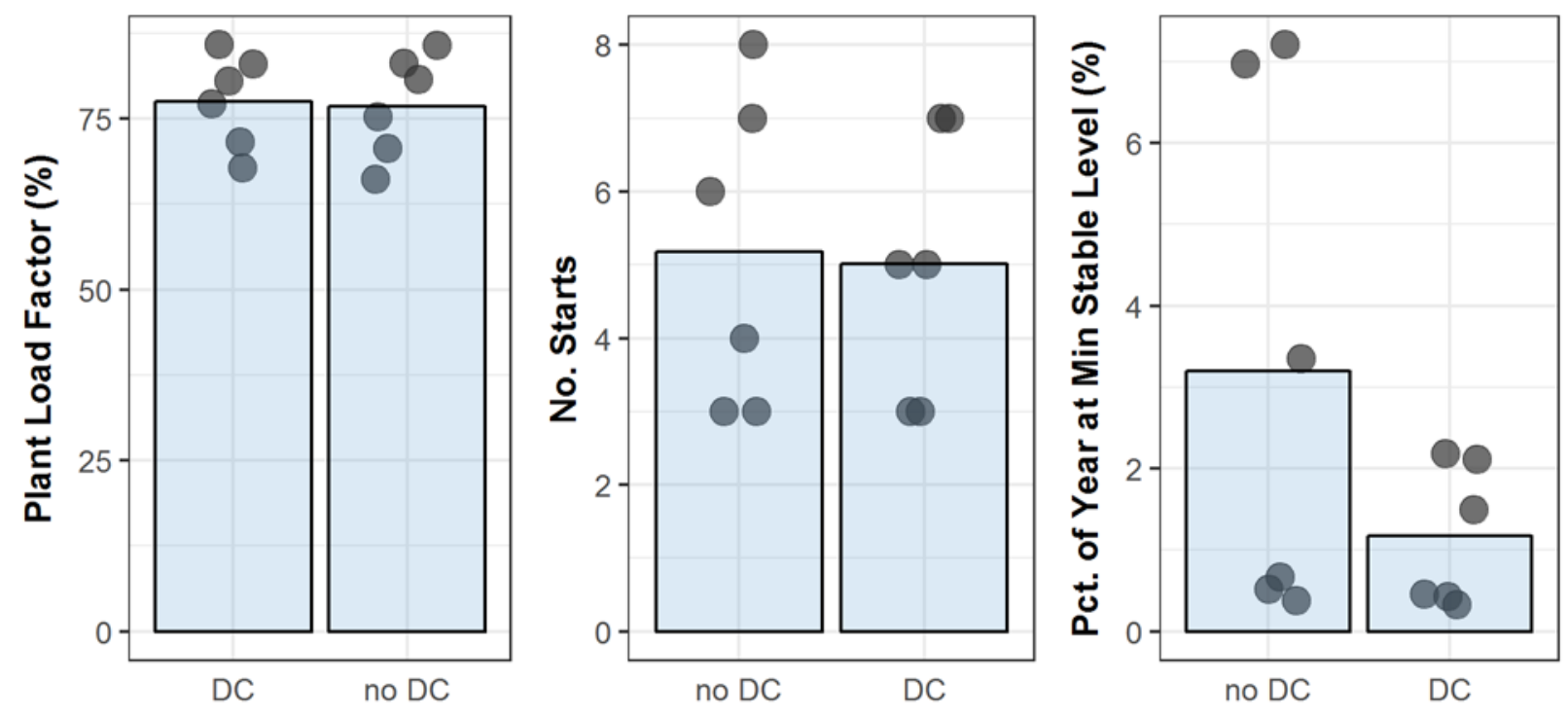

Figure 10. Coal generator operations with and without a DC tie, Sri Lanka.

Note: Black dots represent each of Sri Lanka's 2025 coal generators. Blue bars give the capacity-weighted mean across Sri Lanka's 2025 coal generators.

\footnotetext{
${ }^{10}$ Non-coal thermal is defined as the aggregate of gas, diesel, fuel oil and biomass.
} 

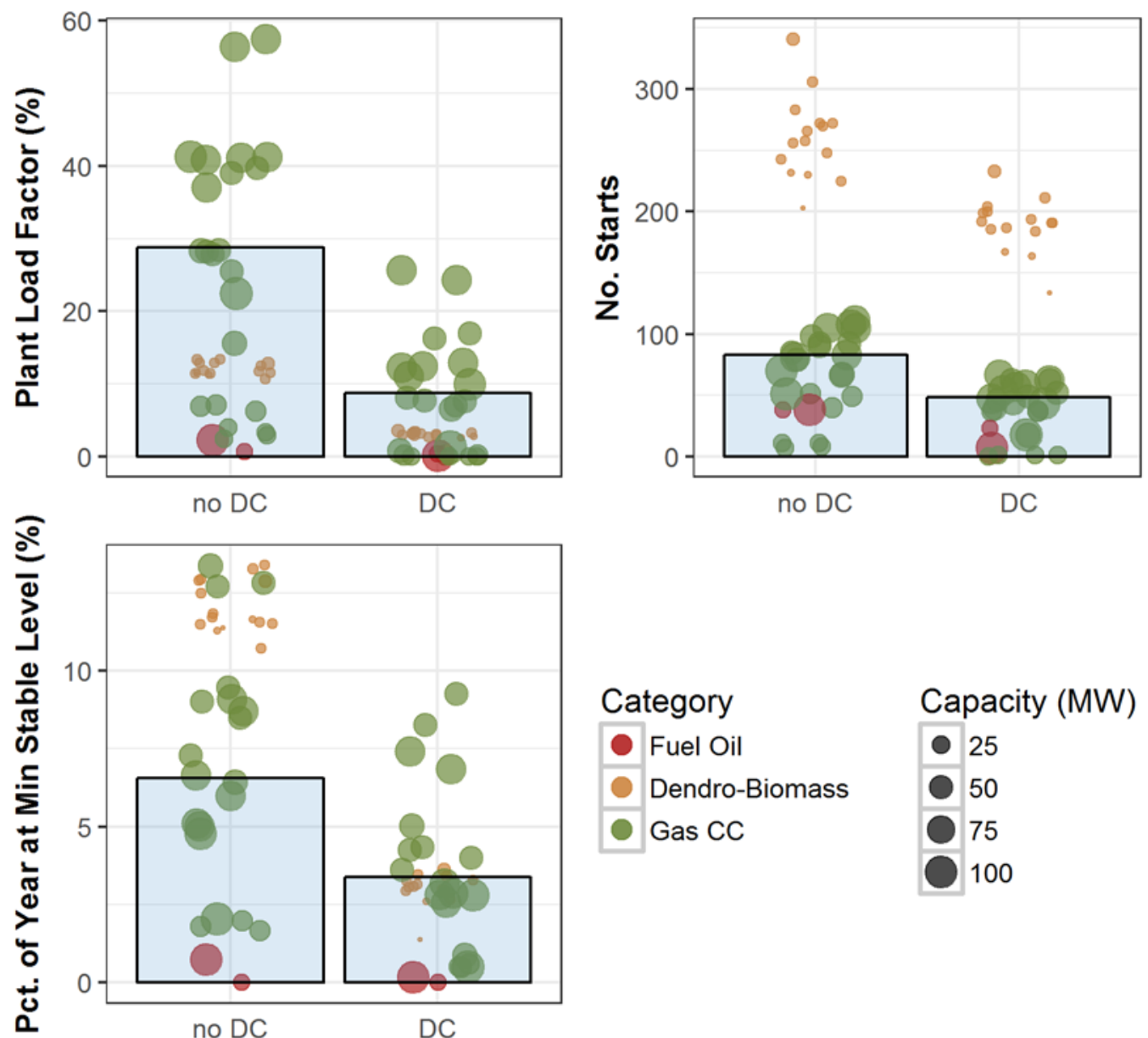

Figure 11. Other thermal generator operations with and without a DC tie, Sri Lanka

Note: Dots represent individual plants sized to nameplate capacity. Blue bars give the capacity-weighted mean.

The combined footprint of Sri Lanka and India's Southern region experiences less demanding net load ramps when compared to each region operating independent of one another.

In power systems, net load refers to the total demand for electricity minus the portion served using variable renewable resources. It is a metric used to track post-RE demand variability, which is typically met from conventional sources. In an independently operated power system, variability in net load must be met by local generators. In interconnected systems, total variability in net load typically falls due to the smoothing effects of small fluctuations over a wider geographic area and the combined system's larger pool of generation resources available to meet changes in net load. Figure 12 compares one-hour net load ramps as a fraction of non-RE online capacity between Sri Lanka, India's Southern region, and the combined Sri Lanka Southern region footprint resulting from the DC tie's addition. Although the difference is modest, Sri Lanka and the Southern region must collectively meet fewer demanding net load ramps when connected by a DC tie. 

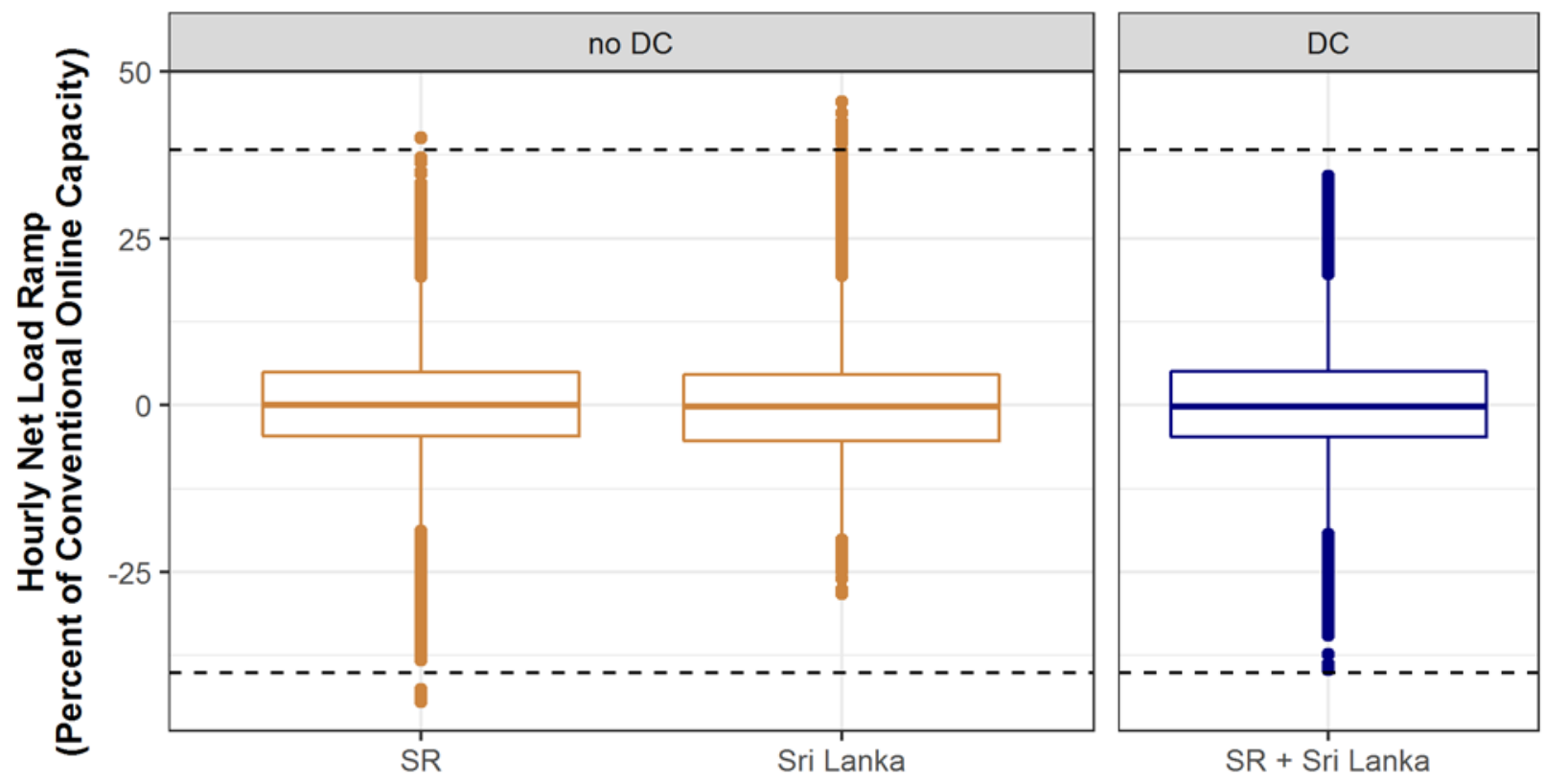

Figure 12. Hourly net load ramp rates as a ratio of non-RE online capacity: Southern region (SR), Sri Lanka and the combined SR + Sri Lanka footprint created by the DC tie.

Note: Boxes represent divisions into 25th percent quantiles, meaning the tail above the box represents $25 \%$ of the ramps, the area inside the box represents the middle $50 \%$, and the tail below the box represents the bottom $25 \%$.

The middle line is the median. The dotted lines represent the maximum up and down net load ramps from the scenario with the DC tie addition.

\subsection{Hydro Fleet Operations}

Large hydro plant operations are optimized across multiple time scales to minimize system costs. On an annual and seasonal time scale, limited (but zero variable cost) hydro generation must be allocated across the year, subject to maximum energy limits and natural inflow constraints. In single-day optimization steps, hydro plants are dispatched during periods when their operation most efficiently displaces expensive marginal thermal generation. The $200 \mathrm{MW}$ pumped storage capacity in 2025 allows Sri Lanka to further boost hydro output during peak load periods at the cost of increased pump load in off-peak hours.

Figure 13 compares average hourly large hydro generation (upper panel) and pumped storage operations (lower panel) with and without a DC tie. Generation from Sri Lanka hydro is displaced during the day by imports from India across the DC tie, allowing more hydro generation during the evening. Since Sri Lanka's average peak demand hour is 7 p.m., increased evening hydro availability can help prevent expensive gas and diesel starts. To support the evening operations, Sri Lanka's pumped storage plant increases its midday loading in the DC tie scenario and generates more during the evening peak hours. 

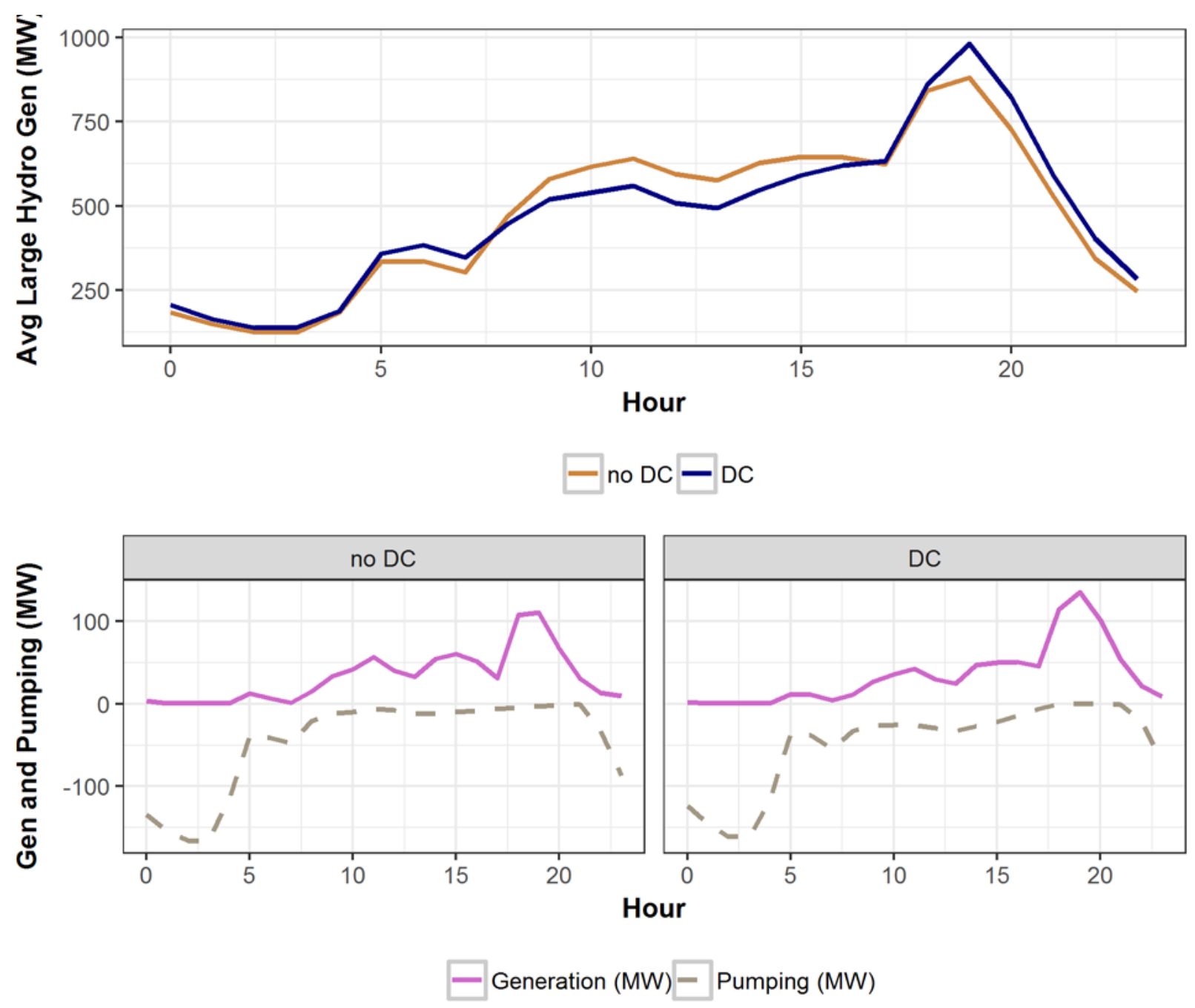

Figure 13. Sri Lanka's average hourly large hydro generation (top panel) and average hourly pumped storage operations (bottom panel), with and without a DC tie

\subsection{Implications for Wind and Solar Integration}

Sri Lanka's 2025 power system, despite its 19.7\% non-conventional RE penetration (excluding pump load), experiences virtually no RE curtailment in simulations with and without the DC tie. Its flexible large hydro and thermal generators can effectively absorb the variability of its 1,856 MW of mini-hydro, wind, utility-scale PV, and rooftop-PV capacity.

India's Southern region however experiences 3.0\% RE curtailment without the DC tie. As discussed in section 3.2, the DC tie can function as an alternative pathway for the Southern region's surplus RE exports. Table 13 compares RE curtailment in Sri Lanka and India, which falls by $8.1 \%$ overall and $8.5 \%$ in India's Southern region with the DC tie. 
Table 13. RE Curtailment in India and Sri Lanka, with and without DC Tie

\begin{tabular}{lllll}
\hline & \multicolumn{2}{l}{ Fraction of Available RE Curtailed (\%) } & \multicolumn{2}{l}{ RE Energy Curtailed (GWh) } \\
\cline { 2 - 5 } & No DC & DC & No DC & DC \\
\hline India & 1.4 & 1.3 & 5300 & 4800 \\
Sri Lanka & 0.0 & 0.0 & 0 & 0 \\
\hline Total & 1.4 & 1.3 & 5300 & 4800 \\
\hline
\end{tabular}

Of India's RE curtailment without the DC tie, $82.8 \%$ occurs in the daytime ( 6 a.m- 6 p.m.) during the monsoon season (June-September). Figure 14, a by-hour comparison of total intertie flows to the total reduction in India's RE curtailment with a DC tie, illustrates the relationship between India's increased daytime exports to Sri Lanka and reduced daytime RE curtailment.

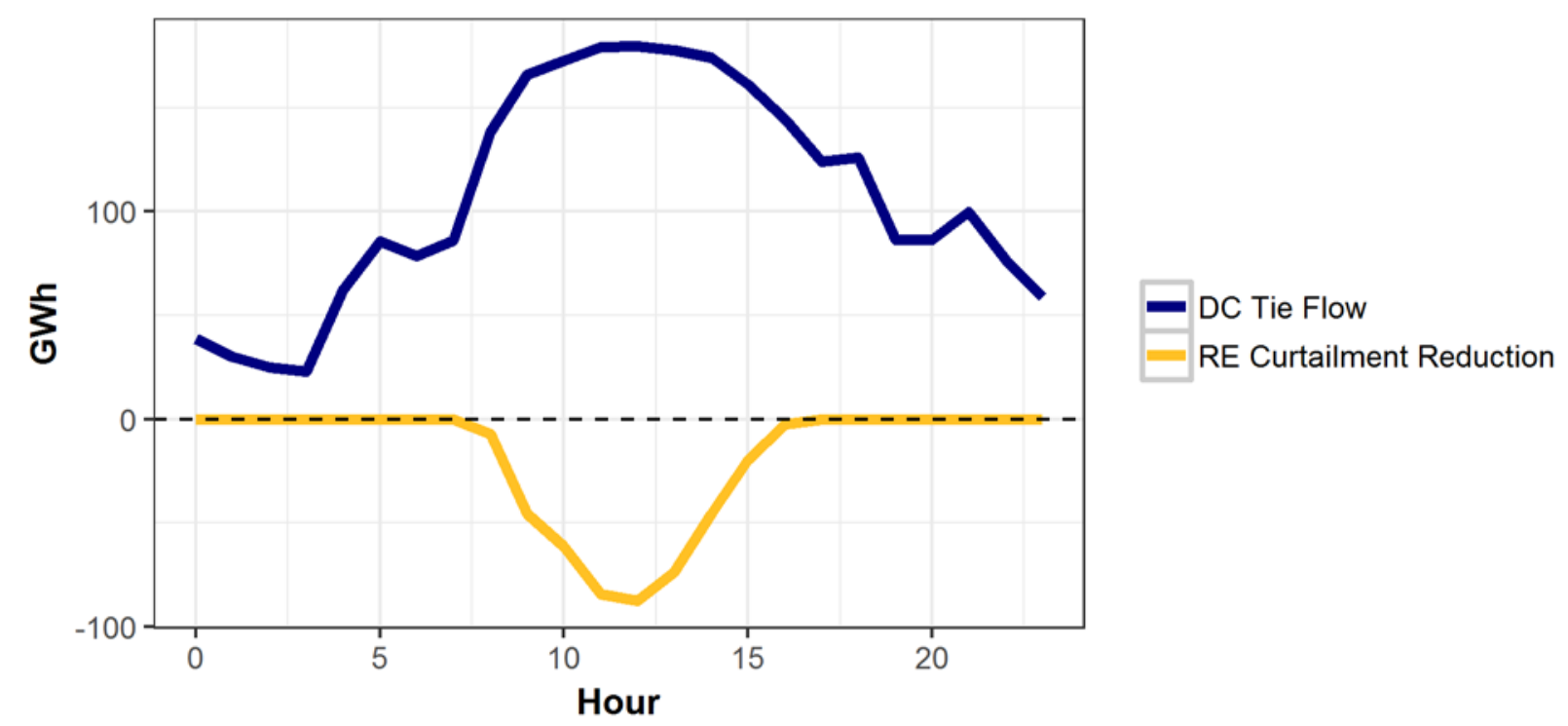

Figure 14. Comparison of total annual intertie flows and RE curtailment reduction in India with a DC tie, by hour.

Note: Positive DC tie flow values indicate energy moving from India to Sri Lanka.

CBET has the potential to aid in renewable energy integration by both reducing RE curtailment inside India and lowering the demands placed on thermal generators in following net load profiles.

\subsection{Tie Utilization}

The following section more closely examines the two countries' use of the DC tie's $500 \mathrm{MW}$ capacity. Figure 15 (A) is a duration curve of power flows across the DC tie throughout the year, and Figure 15 (B) normalizes cross-border flows to Sri Lanka's load. 

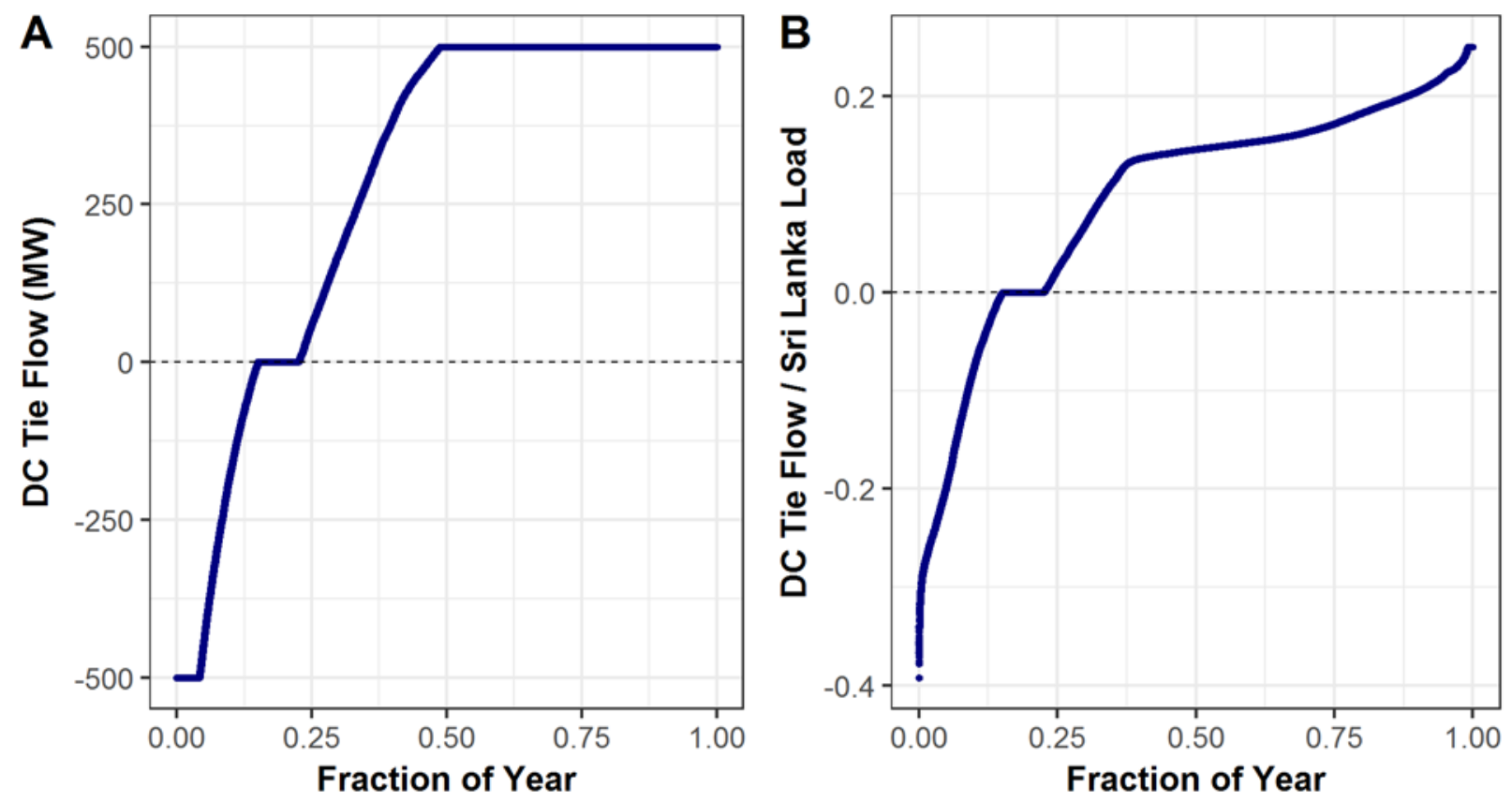

Figure 15. Duration curve of (A) DC tie flows and (B) DC tie flows normalized to Sri Lanka's load.

Note: Positive values indicate energy moving from India to Sri Lanka.

While energy normally flows from India to Sri Lanka across the DC tie, Sri Lanka exports 0.3 TWh to India over $13.8 \%$ of the year. For $8.8 \%$ of the year, energy being produced on either side of the DC tie is close enough in cost that there is no flow across the line.

Energy flows from India to Sri Lanka across the DC tie for $77.4 \%$ of the year, totaling $2.9 \mathrm{TWh}$. However, for $13.8 \%$ of the year Sri Lanka is an exporter, sending $0.3 \mathrm{TWh}$ to India. Roughly one-third of Sri Lanka's exports occur in December, when additional Sri Lanka hydro availability makes its energy less expensive. For $8.8 \%$ of the year, the marginal cost difference between Sri Lanka and India does not exceed the USD 15.38 (INR 1,000) transaction charge placed on the DC tie, and CBET does not occur.

At their instantaneous peak, imports from India meet $25 \%$ of Sri Lanka's load. Imports account for $12.1 \%$ of Sri Lanka's aggregate demand. Sri Lanka's exports to India never exceed $0.4 \%$ of India's load and meet less than $0.1 \%$ of India's aggregate demand.

The DC tie is congested for $57.9 \%$ of the year, implying that transfer capacity beyond 500 MW could provide additional economic benefits.

For 5,060 hours, or $57.9 \%$ of the year, the DC tie is congested: 4,772 hours from India to Sri Lanka and 288 hours from Sri Lanka to India. The frequent congestion implies that the two countries could benefit from transfer capacity beyond $500 \mathrm{MW}$. 


\subsection{Internal Transmission Flows}

The study aggregates in-state transmission in India and thereby assumes that India would build enough transmission in Tamil Nadu to facilitate DC power transfer up to $500 \mathrm{MW}$ to and from Sri Lanka.

The study represents and enforces Sri Lanka's AC transmission network using a DC power flow approximation (see Section 2.3.3). Sri Lanka's network experiences no congestion in the 2025 simulation, with or without a DC tie. Although the results suggest that transmission congestion inside Sri Lanka will not impede CBET with India, an AC power flow representation of Sri Lanka's transmission network would more accurately answer the question of whether Sri Lanka has sufficient internal transmission to always accommodate injections and withdrawals at the DC tie endpoint.

Figure 16 is a visualization of annual energy flows on Sri Lanka's transmission network with and without the DC tie. With a DC tie, more power wheels from North Central through Central to the Western Province as natural gas and other thermal generation in the Western Province declines.

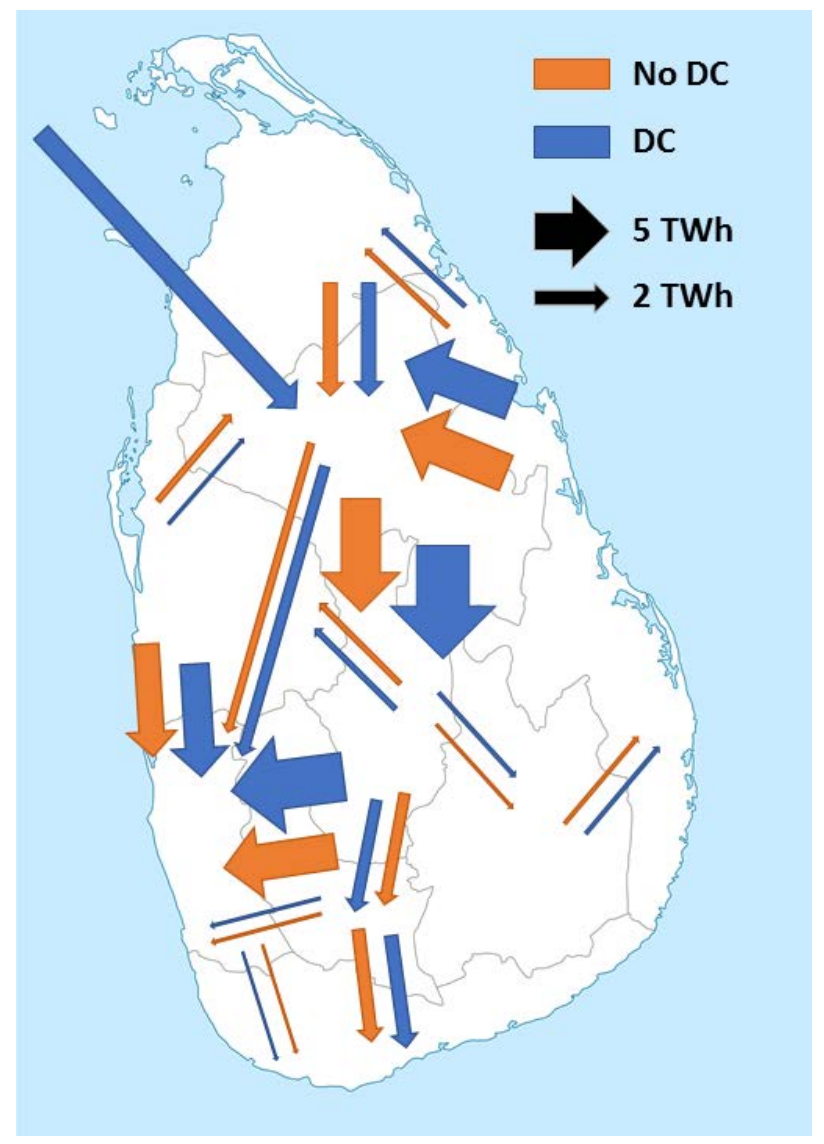

Figure 16. Annual energy flows on Sri Lanka's transmission network with and without the DC tie.

Source: Background image created by AlexR.L. and licensed under a Creative Commons Attribution-Share Alike 3.0 Unported license. Modified image licensed under a Creative Commons Attribution-Share Alike 3.0 Unported license. 


\subsection{Example System Operations}

The following section analyzes the impact of CBET on daily system operations for select days of interest representing varying demand, hydro, and wind and solar generation patterns.

During the dry season, Sri Lanka can meet its load at lower cost through imports.

Figure 17 compares Sri Lanka's dispatch with and without a DC tie on April 10, when its hydro generation is below the annual average and evening peak demand is low relative to daytime demand. While coal generation remains roughly constant, other thermal generation falls by $48.8 \%$, resulting in a $30.5 \%$ reduction in Sri Lanka's production cost for the day. In the DC tie scenario, the deficit between domestic generation and load is met by imports from India.

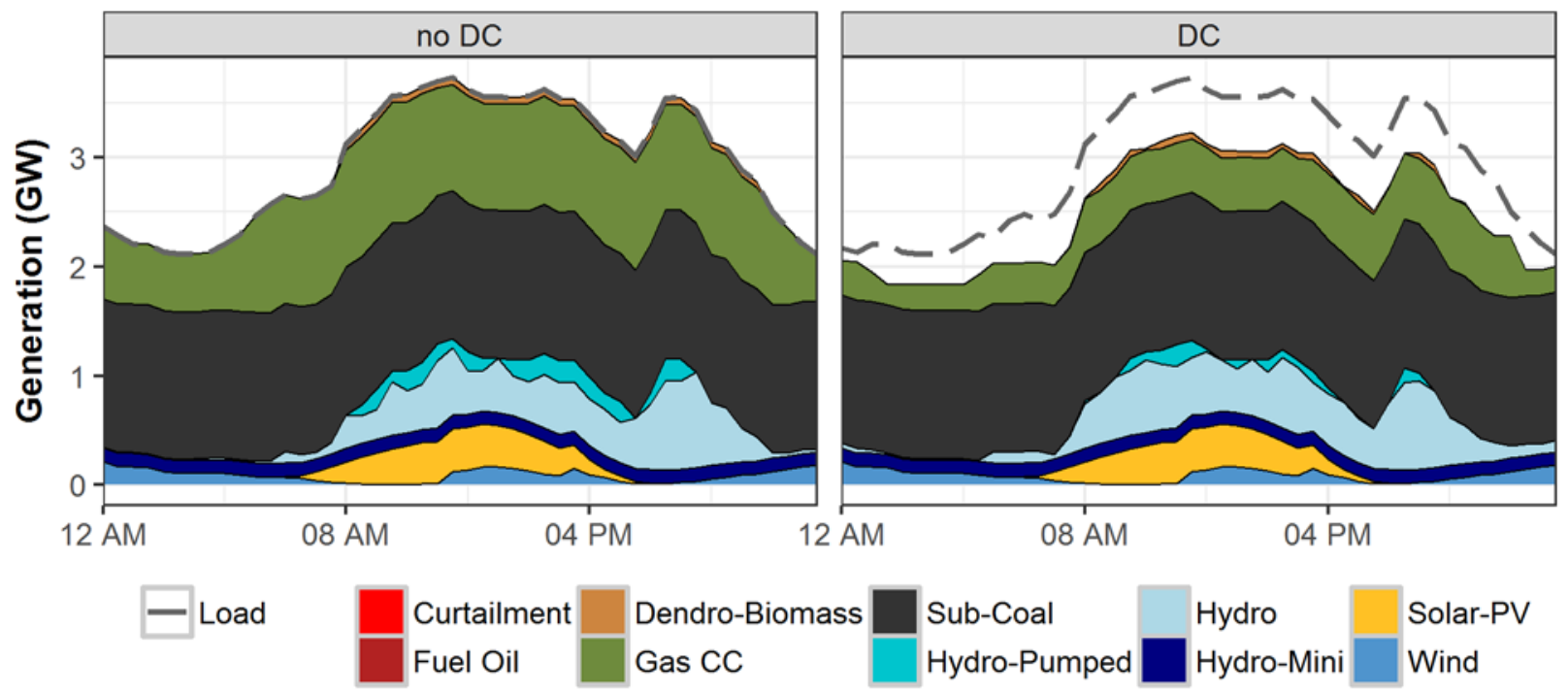

Figure 17. Sri Lanka's April 10 dispatch, with and without the DC tie

Periods of high hydro and coal availability allow Sri Lanka to provide low-cost exports to India.

Figure 18 compares Sri Lanka's dispatch with and without a DC tie on November 30 when hydro availability is relatively high, and the system has higher evening demand relative to daytime demand. Sri Lanka is exporting during periods when generation exceeds demand and importing during periods with deficit generation. Total generation in Sri Lanka rises by $1.5 \%$ with the DC tie as Sri Lanka exports to India at night and imports from India during the peak daytime and evening load hours. With the DC tie, Sri Lanka's hydro generators adjust their dispatch to generate more when Sri Lanka exports and less when it imports. 


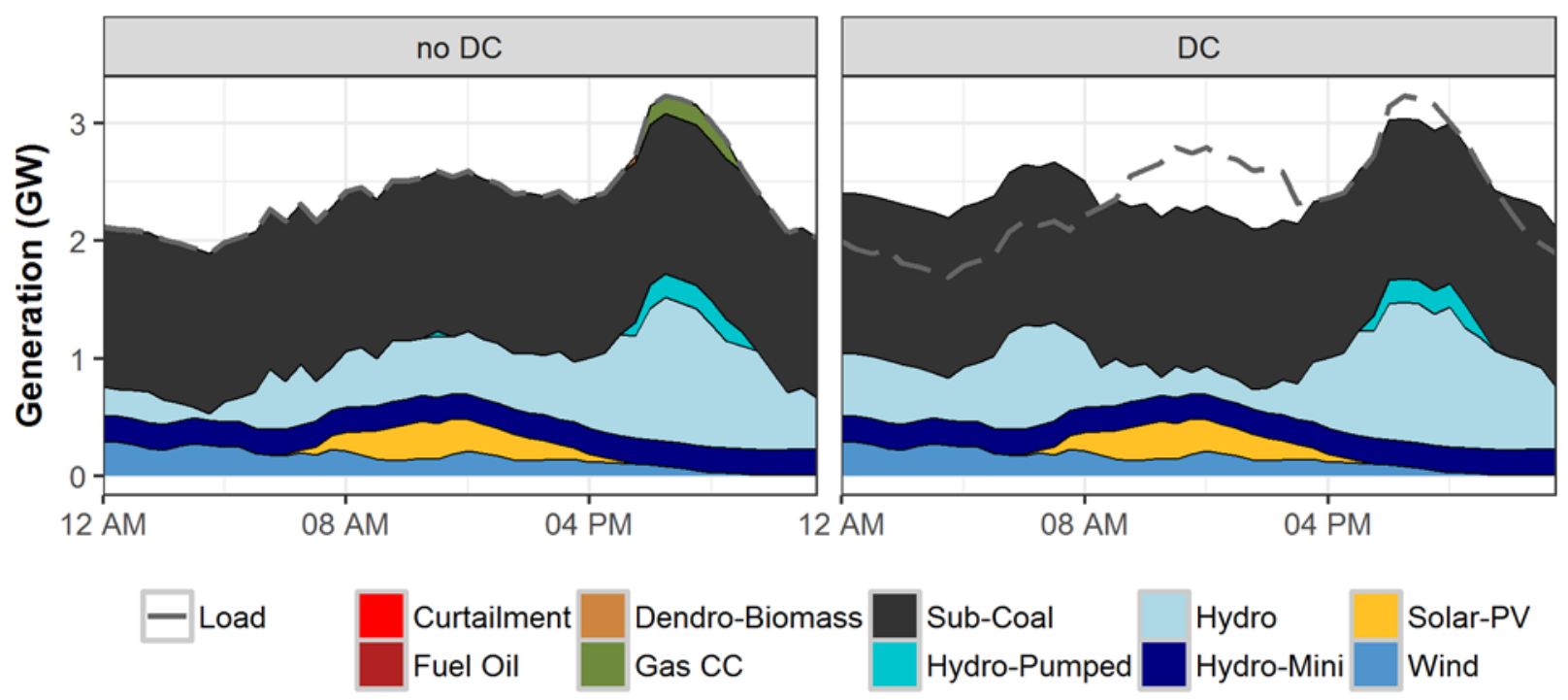

Figure 18. Sri Lanka's November 30 dispatch, with and without the DC tie

Example operations on June 14 illustrate four key impacts of a DC tie on system operations.

Figure 19 shows generator dispatch in Tamil Nadu and Sri Lanka on June 14, which illustrates four key impacts of CBET on system operations. The example of 14 June allows for examination of all four impacts in a single day, but the operational impacts illustrated appear throughout the year. 

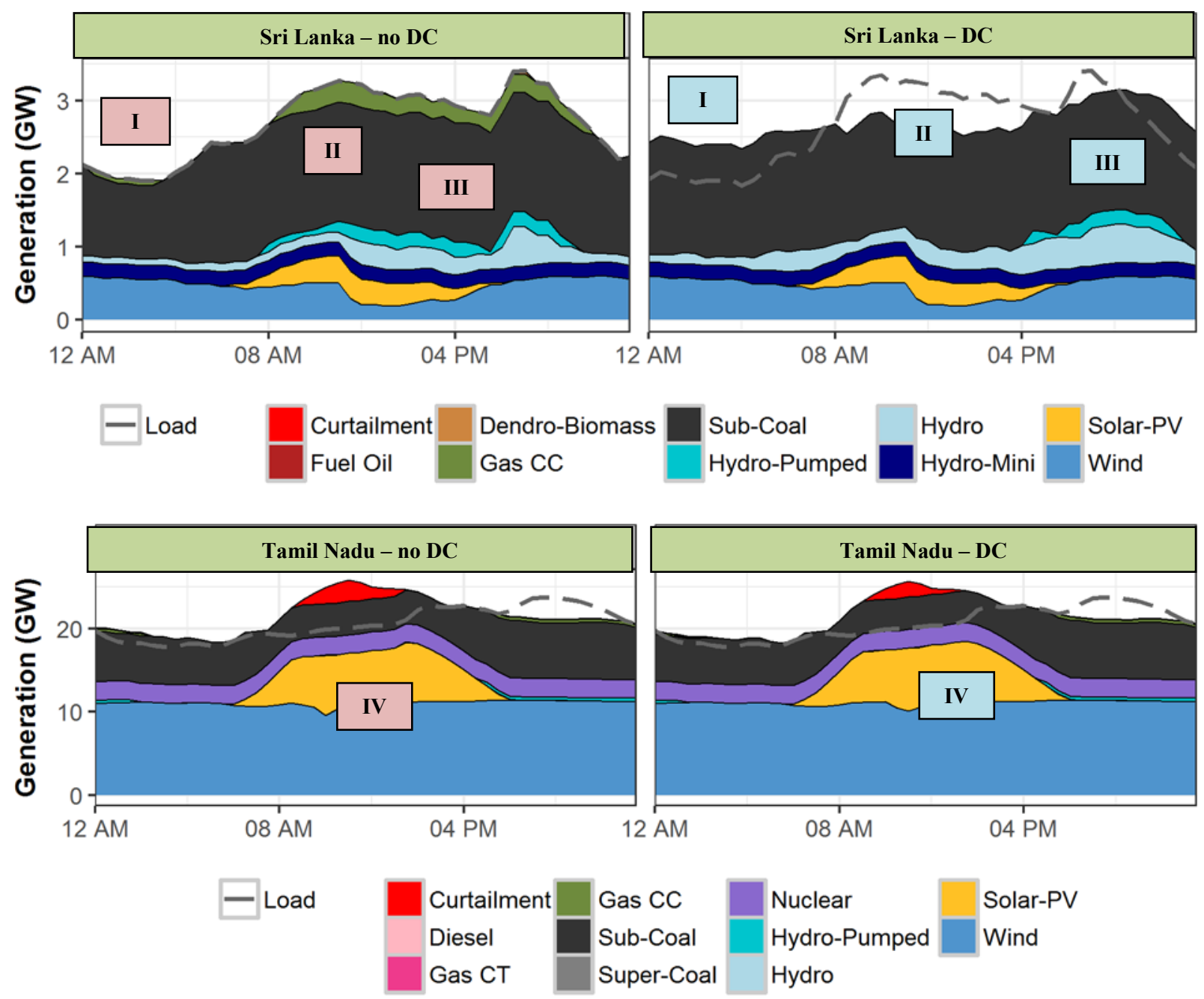

Figure 19. Dispatch in Sri Lanka and Tamil Nadu on June 14, illustrating:

I. Increased efficiency of Sri Lanka coal operations

II. Displacement of Sri Lanka gas generation by imports from India

III. Day to evening shift in Sri Lanka pumped hydro generation

IV. RE curtailment reduction in India's Southern region

\section{Increased efficiency of Sri Lanka coal operations}

In the early morning hours on 14 June in the no DC scenario, low load in Sri Lanka forces coal generators to ramp down and operate inefficiently at their minimum stable levels, where heat rates are higher. With a DC tie, exports of over $3 \mathrm{GWh}$ between midnight and 7:30 allow coal generators to increase output and operate at more efficient heat rates. Annually, Sri Lanka pays $0.2 \%$ less per MWh for its coal generation with a DC tie, reflecting the more efficient plant utilization. While modest, the cost savings reflect a decrease in the stress placed on the coal fleet, which could lead to lower outage rates and longer plant lifetimes (Kumar et al. 2012). However, 
as discussed in section 3.3, gas and other thermal generators in Sri Lanka experience more starts and time at minimum stable level relative to time on in the DC tie scenario.

\section{Displacement of Sri Lanka gas generation by imports from India}

Three Sri Lankan combined cycle gas units (405 MW total) do not turn on, saving over USD 100,000 in start costs and over USD 250,000 in variable operational costs. Imports from India, mainly midday, totaling $4 \mathrm{GWh}$ more than offset the reduced generation. Annually, gas generation in Sri Lanka falls by $69.2 \%$, while other non-coal thermal generation falls $78.2 \%$.

III

Day to evening shift in Sri Lanka pumped hydro generation

Sri Lanka's highest daily load hours are typically from 10-12 and 18-19. Without a DC tie, Sri Lanka's large hydro plants and the planned $200 \mathrm{MW}$ pumped storage plant typically concentrate generation during these high load periods (see Figure 13). However, because imports from India over the DC tie displace some of Sri Lanka's daytime generation, Sri Lanka's hydro is able to focus generation during the evening peak, when its value to the system is highest. On 14 June, the planned $200 \mathrm{MW}$ pumped storage plant uses imports from India to charge slightly more than 1 GWh between midnight and 10:00, before generating $1 \mathrm{GWh}$ starting at 16:30 and continuing through the day's end..

\section{RE curtailment reduction in India's Southern region}

The 500 MW DC tie to Sri Lanka provides an export option for generation in India's Southern region, which because of operational constraints, must curtail some of its renewable energy output. On 14 June, India exports $4 \mathrm{GWh}$ of energy to Sri Lanka, allowing for the evacuation of approximately $3 \mathrm{GWh}$ of previously curtailed wind and solar generation in Tamil Nadu. Annually, with a DC tie the Indian states of Tamil Nadu, Andhra Pradesh and Karnataka experience the largest reductions in RE curtailment — approximately $300 \mathrm{GWh}(22 \%), 100 \mathrm{GWh}$ $(5 \%)$ and $50 \mathrm{GWh}(3 \%)$, respectively. 


\section{Hydro Sensitivity Results}

In the following section we expand the results presented in Section 3 to analyze how the operational impacts of CBET may change under different assumptions about hydropower availability in Sri Lanka. We tested dry and wet hydro sensitivities to capture the change in hydro availability. For information on the definition and design of hydro sensitivities, see Section 2.

\subsection{Electricity Production Cost}

Compared to a medium hydro year, total production cost savings from CBET are $17 \%$ higher in a dry year and $11 \%$ lower in a wet year.

Production cost savings from CBET increase as available hydropower in Sri Lanka decreases. In a dry hydro year, Sri Lanka must rely more on relatively expensive thermal generation from gas and fuel oil plants to meet its load. The DC tie enables lower cost imports from India to displace this thermal generation, reducing the annual operating cost for the combined system by USD 210 million. In a wet year, there is less thermal generation in Sri Lanka that can be displaced by imports and the total savings fall slightly to USD 160 million. Table 14 compares the individual and combined variable cost for India and Sri Lanka (in millions of USD) for the three hydro sensitivities.

Table 14. Electricity Production Cost with and without DC Tie, India and Sri Lanka, Hydro Sensitivities

\begin{tabular}{llllllllll}
\hline & \multicolumn{2}{l}{ No DC $(\mathbf{\$ M})$} & \multicolumn{3}{l}{ DC $(\mathbf{\$ M})$} & \multicolumn{3}{c}{ Change in Costs (\%) } \\
\cline { 2 - 10 } & Dry & Med & Wet & Dry & Med & Wet & Dry & Med & Wet \\
\hline India & 34,440 & 34,430 & 34,430 & 34,510 & 34,480 & 34,460 & 0.2 & 0.2 & 0.1 \\
Sri Lanka & 780 & 690 & 610 & 500 & 450 & 420 & -36.6 & -34.6 & -31.5 \\
\hline Total & 35,220 & 35,120 & 35,040 & 35,010 & 34,940 & 34,880 & -0.6 & -0.5 & -0.5 \\
\hline
\end{tabular}

In general, the production cost savings are largely due to reduced fuel and variable operation and maintenance costs. Fewer plant starts account for $6 \%$ of total savings in the dry sensitivity, $8 \%$ in the medium sensitivity, and $12 \%$ in the wet sensitivity, a disproportionately high contribution to overall savings considering start costs account for only $1.3 \%$ of total costs.

\subsection{Electricity Generation by Resource}

Figure 20 summarizes the change in annual generation with the DC tie by resource type across the India regions and Sri Lanka. In all hydro scenarios, CBET results in lower generation from plants in Sri Lanka and increased output from plants in India. While most of the increase in India occurs in the Southern and Western regions, which are geographically closest to Sri Lanka, every region exhibits some changes in annual generation with the DC tie.

The change in generation across all regions is highest in a dry year when the opportunity to displace gas, fuel oil, and dendro biomass plants in Sri Lanka with lower-cost coal generation from India is highest. In a wet year, generation from more efficient coal plants in Sri Lanka 
displaces output from less efficient units in India. Sri Lanka's coal generation increases by 3\% with the DC tie compared to a wet year with no interconnection.
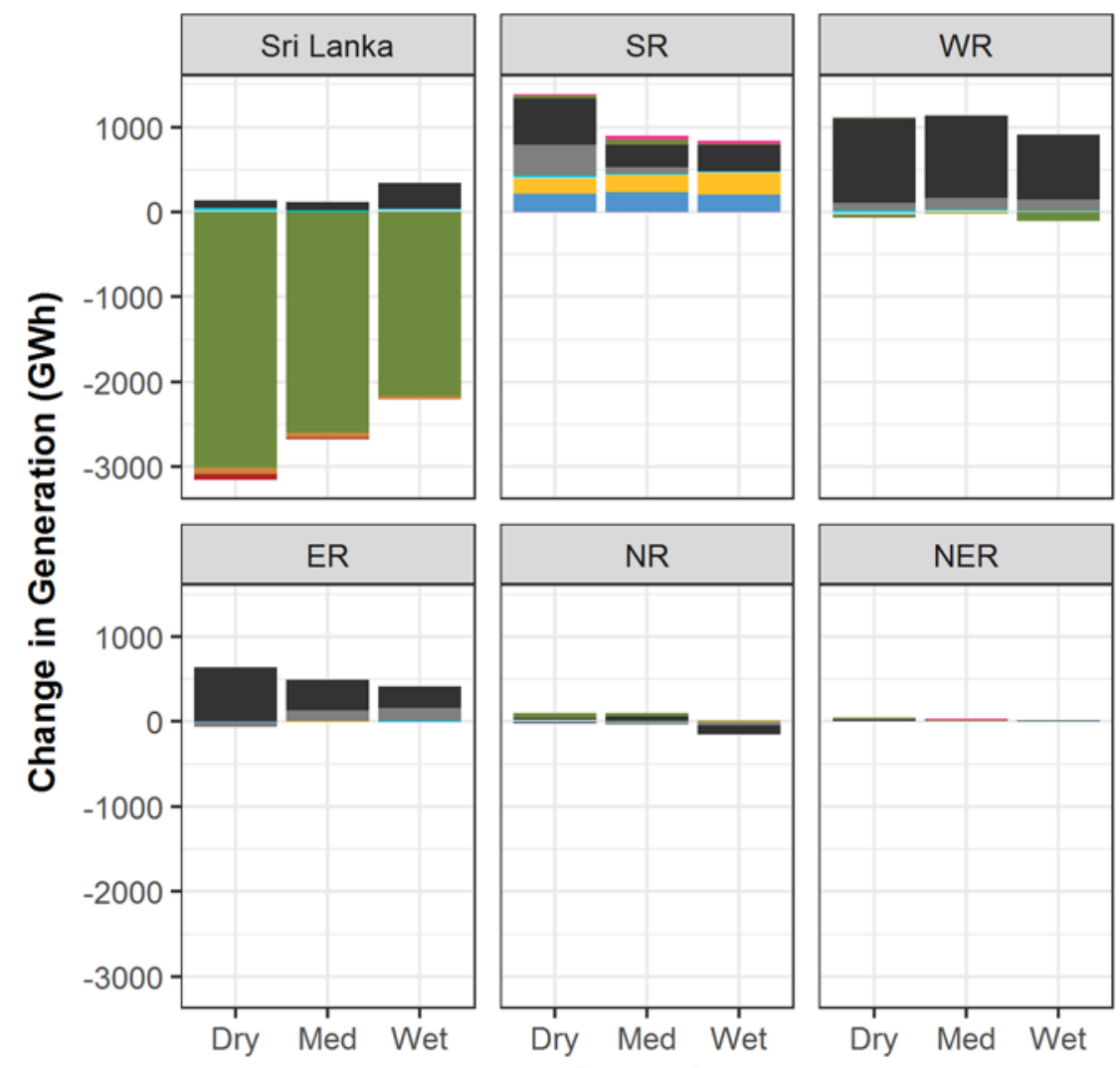

Other
Fuel Oil
Diesel
Dendro-Biomass
Gas CT
Gas CC
Sub-Coal
Super-Coal
Nuclear
Hydro-Pumped
Hydro
Solar-PV
Wind

Figure 20. Change in generation with the DC tie by resource type and region for each hydro sensitivity.

Note: India regions: SR - Southern region; WR - Western region; ER - Eastern region; NR - Northern region; NER Northeastern region.

\section{A dry Sri Lankan hydro year results in 540 GWh more coal generation in India, but has little effect on RE curtailment.}

Section 3 notes that CBET reduces RE curtailment in India. This result is impacted little by the availability of hydro in Sri Lanka because congestion on the DC tie typically limits imports from India in high RE curtailment periods. Instead, increased India coal generation makes up for the reduced output from Sri Lanka hydro.

\subsection{Tie Utilization}

As discussed in Section 3, the full capacity of the DC line is used for large portions of the year in a medium hydro year. As hydro availability in Sri Lanka decreases, imports from India (and network congestion) increase, from $2.5 \mathrm{TWh}$ in the wet sensitivity to $3.2 \mathrm{TWh}$ in the dry sensitivity. In the wet sensitivity, Sri Lanka has increased opportunities to export power, particularly during off-peak hours in wetter months later in the year. Total exports increase by $99 \%$ compared to a medium hydro year. Figure 21 shows the hourly patterns of trade between India and Sri Lanka for an average day in each month and hydro sensitivity. 

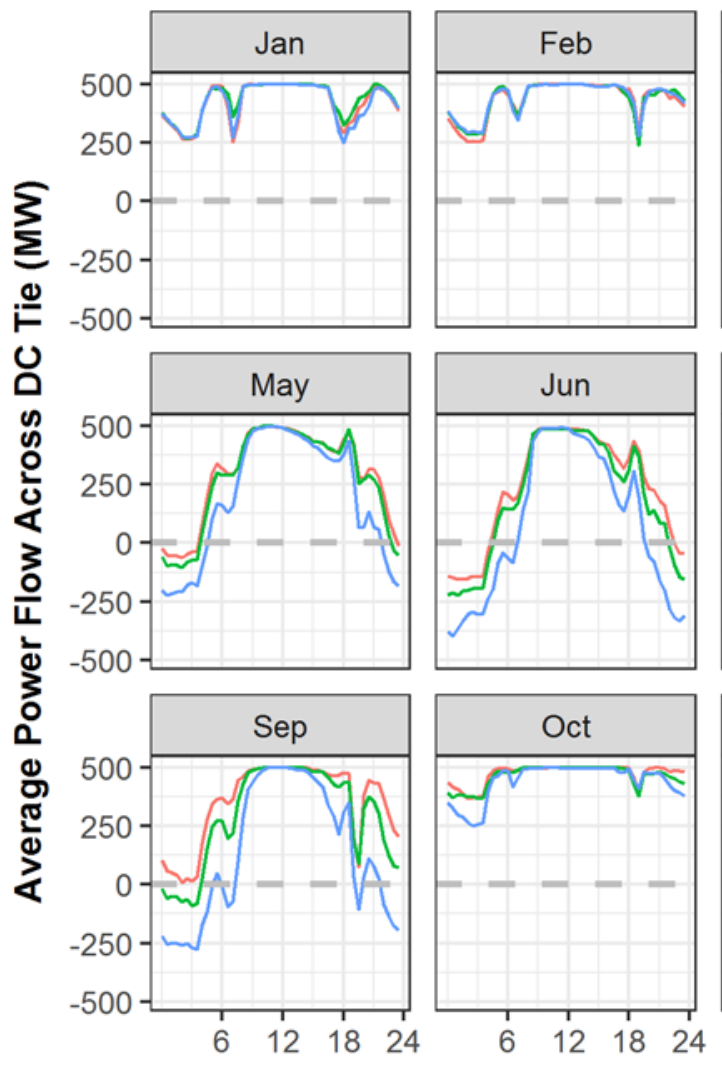

Hour
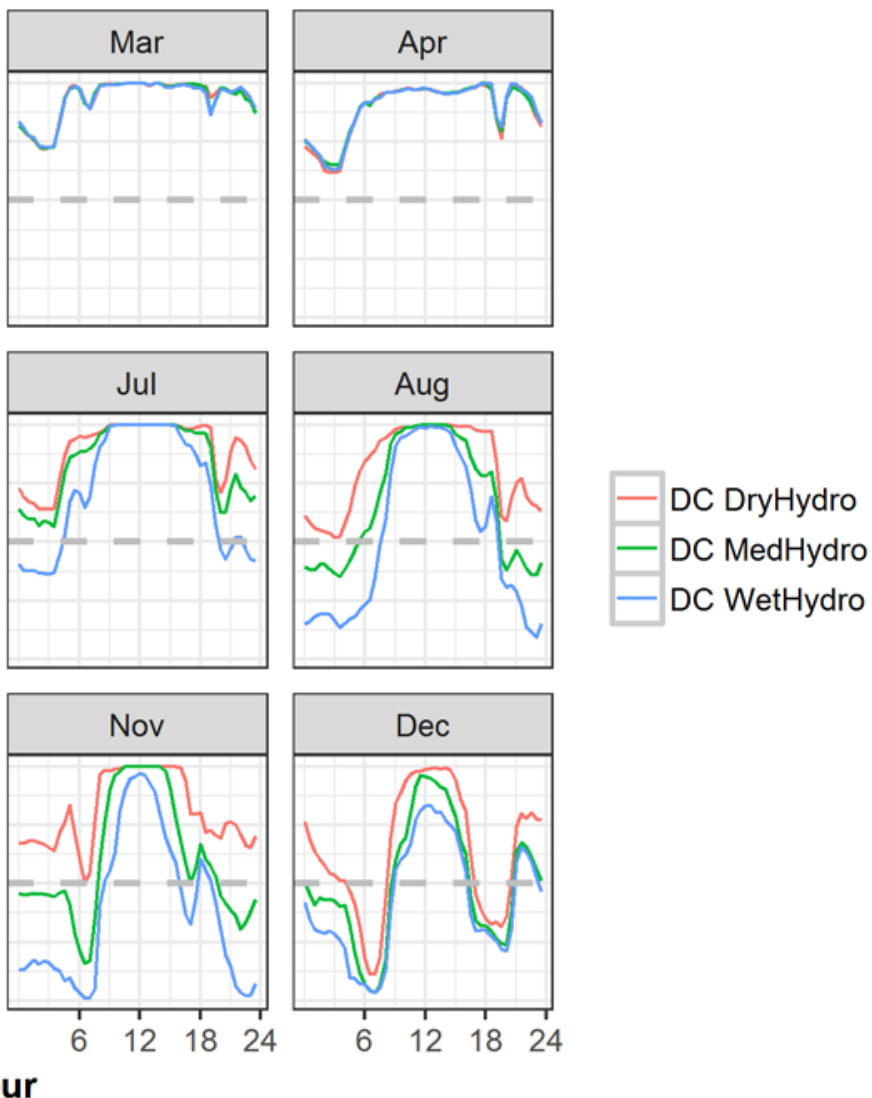

Figure 21. Daily and seasonal patterns of trade between India and Sri Lanka for all hydro sensitivities.

Note: Positive values indicate energy moving from India to Sri Lanka.

In each month, Sri Lanka's imports peak during the middle of the day when both local demand and solar output in India are high. Sri Lanka's internal AC network, which effectively managed imports and exports under medium hydro conditions continues to do so in the wet and dry hydro sensitivities.

During wet years, Sri Lanka has increased opportunities to export to India during the evenings and wet season.

In the wet-year sensitivity, further trade is restricted by the transfer capacity of the DC tie in $54 \%$ of periods. This increases to $62 \%$ of periods during a dry year.

\subsection{Security of Supply}

Interannual variability in hydropower resources presents a possible threat to security of supply for Sri Lanka's power system. This section considers the impact of increased CBET on strategic, operational, and national security of supply in Sri Lanka. This analysis considers only the impacts of varying hydro availability and does not consider other sources of uncertainty such as changes in fuel prices or contingency events. A complete analysis of security of supply impacts in both India and Sri Lanka is beyond the scope of this study. This section aims to highlight key findings and does not attempt to evaluate or prescribe acceptable levels of security of supply. 
Because of the relative sizes of the two systems, impacts of varying Sri Lanka hydro availability on security of supply in India are minor.

Cross-border trade increases strategic security of supply in Sri Lanka and can decrease operational risks, but also raises new considerations about operational security of supply and reliance on imports.

\subsubsection{Strategic Security of Supply}

Strategic security of supply refers to longer-term concerns related to the ability of the power system to provide affordable and reliable electricity in the face of events such as droughts. The cost of electricity supply in Sri Lanka is highly sensitive to the availability of hydropower. In a dry hydro year with no DC tie, total generation costs increase $13 \%$ compared to a medium hydro year as more expensive thermal plants are required to meet demand. Through trade with India, Sri Lanka can modestly reduce its cost sensitivity to hydro availability. Assuming the cost of imports is set by the short-run marginal cost in Tamil Nadu, the cost increase in Sri Lanka during a dry year falls from $13 \%$ to $12 \%$. Hydro conditions also impact Sri Lanka's ability to maintain adequate operating reserves, set at $5 \%$ of load in every hour. With no DC tie, unmet operating reserves increase from $400 \mathrm{MWh}$ in a medium year to $450 \mathrm{MWh}$ in a dry year. With the DC tie, there are only $60 \mathrm{MWh}$ of unmet operating reserves in a dry year and $50 \mathrm{MWh}$ in a medium and wet year. It should be noted that these estimates of unmet operating reserves represent a small fraction-less than $1 \%$ - of total operating reserves held throughout the year.

\subsubsection{Operational Security of Supply}

Operational security of supply is related to the ability of the system to respond to contingency events such as unexpected plant or transmission line outages. The ability to trade with India could improve grid resiliency in Sri Lanka during contingency events. In the dry hydro year with no DC tie, there is a one-week period in March when Sri Lanka generators are not able to meet demand due to limited water available for hydro generation and simulated unplanned outages at large coal units. With the DC tie, this demand is met by imports from India, avoiding potential load shedding. The ability to provide a coordinated response during contingency events can only be realized if system operators on both sides of the interconnection are able to adjust scheduled power flows to meet real time conditions. However, as discussed in Section 4.4.3 below, imports across the DC tie often supply more than 14\% of Sri Lanka's instantaneous demand in 2025, creating a new contingency risk if there is a technical failure on the line.

\subsubsection{National Security of Supply}

National security of supply, a concern specific to CBET, is related to the willingness of a country to rely on external agents for their electricity supply. With no non-technical barriers to trade, Sri Lanka could potentially rely heavily on imports to meet domestic electricity needs. Table 15 presents the total amount each country imports across the DC tie each year in energy and as a fraction of that country's annual load. 
Table 15. Annual Energy Imports (TWh) and Fraction of Annual Load Met by Imports (\%) in Each Hydro Sensitivity

\begin{tabular}{llll}
\hline & Dry & Med & Wet \\
\hline \multirow{2}{*}{ Sri Lanka } & 3.2 & 2.9 & 2.5 \\
& $(14 \%)$ & $(12 \%)$ & $(11 \%)$ \\
India & 0.2 & 0.3 & 0.6 \\
& $(0.01 \%)$ & $(0.02 \%)$ & $(0.04 \%)$
\end{tabular}

In a dry hydro year, Sri Lanka imports $3.2 \mathrm{TWh}$, accounting for $14 \%$ of its annual electricity demand. In a wet year when more domestic hydro generation is available, energy imports still account for $11 \%$ of annual load or $2.5 \mathrm{TWh}$. These values represent annual totals; the amount of load met by imports in any one period can be as high as $25 \%$. Figure 22 shows the fraction of load met by imports in Sri Lanka for each period of the year.

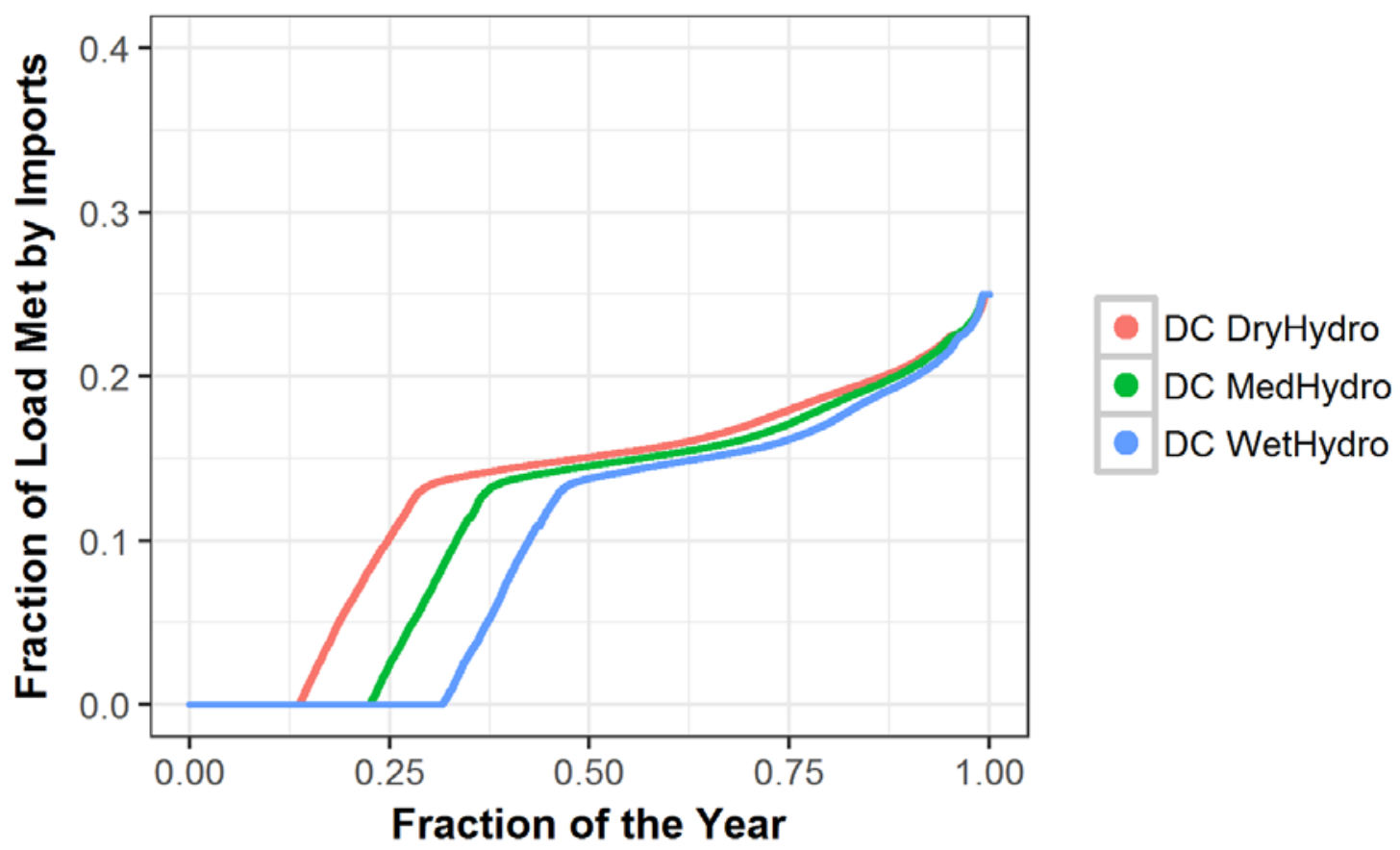

Figure 22. Fraction of annual load met by imports every period in Sri Lanka

For half the year, imports account for more than $14 \%$ of demand in a wet year and $15 \%$ of demand in a dry year. The maximum instantaneous load met by imports is $25 \%$ in all scenarios, constrained by Sri Lanka's operating rule that no single unit, including interconnection lines, can provide more than $25 \%$ of load in any period. In addition to supply risks posed by a technical failure of the DC tie, the level of imports may pose a geopolitical supply risk for Sri Lanka. 


\section{Conclusions}

This study finds that CBET between India and Sri Lanka in 2025 presents an opportunity to reduce the cost of providing electricity, reduce RE curtailment in southern India, and improve system reliability through coordinated use of energy resources.

\section{A DC tie results in combined annual production cost savings of USD 180 million.}

Production cost across India and Sri Lanka falls USD 180 million (0.5\%) in 2025 with a DC tie. Reduced start costs make up a small but disproportionate fraction of the production cost savings (7.6\%), reflecting modestly less stressful thermal generator operations with the DC tie. Most of the savings are due to increased coal generation in India displacing generation from relatively more expensive gas and fuel oil plants in Sri Lanka. Total savings are sensitive to hydro availability in Sri Lanka, falling to USD 160 million (0.5\%) in the wet year sensitivity when there is less thermal generation to displace with imports. In contrast, total savings increase to USD 210 million $(0.6 \%)$ in the dry year sensitivity.

\subsection{TWh of imports from India can displace $69 \%$ of Sri Lanka's natural gas generation.}

The DC tie allows the combined India and Sri Lanka dispatch to more efficiently make use of lower marginal cost resources. Coal and RE generation in India increase by $2.0 \mathrm{TWh}$ and 0.4 TWh, respectively, displacing higher cost fossil-fuel based plants in Sri Lanka. Gas generation in Sri Lanka, which accounts for $15.7 \%$ of that country's generation without a DC tie, falls by $69.2 \%$ when CBET is allowed. Sri Lanka also exports $0.3 \mathrm{TWh}$ to India across $13.8 \%$ of the year, primarily when high domestic coal and hydro availability correlate with low India $b$ wind and solar availability.

\section{Daytime exports to Sri Lanka can reduce RE curtailment by 400 GWh in India's Southern region.}

Because of constraints related to its interconnectedness with other regions, thermal plant flexibility, and high RE penetration relative to load, India's Southern region is more susceptible to RE curtailment than other regions (Palchak et al. 2017b). A DC tie to Sri Lanka provides an additional avenue for daytime energy exports, reducing RE curtailment in the Southern region by $400 \mathrm{GWh}(8.5 \%)$. The reduction in the Southern region's RE curtailment remains unaffected in the wet and dry hydro year sensitivities.

By using the DC tie as a source of flexibility, Sri Lanka sees modestly more efficient coal operations. However, its gas generators, largely displaced by imports, must continue to meet peak load.

With the DC tie, the per-MWh cost of coal generation in Sri Lanka falls $0.2 \%$ because of a modest reduction in the number of hours operating at minimum stable level. However, because Sri Lanka's non-coal thermal generators must continue to cover peak load even as DC tie imports reduce their average PLF from $28.7 \%$ to $8.8 \%$, they experience an $3.8 \%$ increase in perMWh production cost. India's generator operations experience only minor changes at a fleetwide level because of India's large system size relative to Sri Lanka and the $500 \mathrm{MW}$ transfer capacity. 
Daytime imports from India shift Sri Lanka's hydro generation towards the evening. Because imports from India increase available energy during the day, Sri Lanka shifts large hydro generation towards its evening peak load. Its $200 \mathrm{MW}$ pumped storage generator behaves similarly: with a DC tie it pumps more energy during the day and increases its output in the evening.

The DC tie overall improves security of supply in Sri Lanka, dependent upon coordination and cooperation between the two countries.

Interannual variability in hydropower resources creates a security of supply risk for Sri Lanka's power system. Cross-border trade reduces this risk through both decreased costs and increased availability of operating reserves. However, the DC tie also creates new operational and national security risks. While the DC tie can help address contingencies in Sri Lanka, this service is dependent on coordinated response between the countries. The DC tie also helps Sri Lanka meet $12 \%$ of its annual load in the medium hydro year, raising Sri Lanka's reliance on imports. 


\section{References}

CEA (Central Electricity Authority). 2017a. "All India Installed Capacity (in MW) of Power Stations (As on 31.12.2017) Revised." http://www.cea.nic.in/reports/monthly/installedcapacity/2017/installed_capacity-12.pdf.

CEA (Central Electricity Authority). 2017b. "Report on $19^{\text {th }}$ Electric Power Survey of India Volume I."

CEA (Central Electricity Authority). 2018. "National Electricity Plan (Volume I) Generation."

CEB (Ceylon Electricity Board). 2015. "2015 Annual Report." http://www.ceb.lk/index.php?aam_media=34836.

CEB (Ceylon Electricity Board). 2017. Long Term Generation Expansion Plan 2018-2037 (Draft). http://www.pucsl.gov.lk/english/wp-content/uploads/2017/05/LTGEP 2018-2037.pdf.

Kumar N., P. Besuner, S. Lefton, D. Agan and D. Hilleman. 2012. "Power plant cycling costs." Golden, CO: National Renewable Energy Laboratory. NREL/SR-5500-55433. https://www.nrel.gov/docs/fy12osti/55433.pdf.

Milligan M., E. Ela, D. Lew, D. Corbus, Y. Wan, and B.M. Hodge. 2012. "Assessment of Simulated Wind Data Requirements for Wind Integration Studies." IEEE Transactions on Sustainability 3(4):620-626.

Palchak D., J. Cochran, A. Ehlen, B. McBennett, M. Milligan, I. Chernyakhovskiy, R. Deshmukh, et al. 2017a. "Greening the Grid: Pathways to Integrate 175 Gigawatts of Renewable Energy into India's Electric Grid, Vol. I-National Study.” National Renewable Energy Laboratory (NREL), Lawrence Berkeley National Laboratory (Berkeley Lab), Power System Operation Corporation Limited (POSOCO) and U.S. Agency for International Development (USAID).

Palchak D., J. Cochran J., A. Ehlen, B. McBennett, M. Milligan, I. Chernyakhovskiy, R. Deshmukh, et al. 2017b. "Greening the Grid: Pathways to Integrate 175 Gigawatts of Renewable Energy into India's Electric Grid, Vol. II—Regional Study,” National Renewable Energy Laboratory (NREL), Lawrence Berkeley National Laboratory (Berkeley Lab), Power System Operation Corporation Limited (POSOCO) and U.S. Agency for International Development (USAID).

Singh, A., J. Parikh, K. Agrawal, D. Khare, R. Panda, P. Mohla. 2013 "Prospects for Regional Cooperation on Cross-Border Electricity Trade in South Asia," U.S. Agency for International Development (USAID), Integrated Research and Action for Development (IRADe). https://sarienergy.org/wp-content/uploads/2016/03/Prospects-for-Regional-Cooperation-CBET-2013.pdf

Tayal, H.L., S. Ghose, F. Rienzie. 2002. "Viability of Developing a Transmission System Interconnection between India and Sri Lanka: Technical Options and Investment Requirements." Prepared for USAID-SARI Energy Program by Nexant. 
Timilsina, G., M. Toman. 2016. "Potential gains from expanding regional electricity trade in South Asia." Energy Economics 60: 6-14.

WECC (Western Electricity Coordinating Council), “Transmission expansion planning home and GridView WECC database," Western Electricity Coordinating Council, Salt Lake City, UT, USA. https://www.wecc.biz/Reliability/2024-Common-Case.zip.

Wijayatunga, P., D. Chattopadhyay. P. Fernando. 2015. "Cross-Border Power Trading in South Asia: A Techno Economic Rationale.” ADB South Asia Working Paper Series No. 38. Manilla: Asian Development Bank (ADB).

https://www.adb.org/sites/default/files/publication/173198/south-asia-wp-038.pdf. 


\section{Appendix A. 2014 Validation}

We validated the 2014 Sri Lanka UC\&ED model using plant-wise 2014 generation data provided by CEB. The following figures compare plant-wise CEB data to model outputs to give an idea of how well the production cost model captures actual system operations.

Figure 23 compares monthly generation by resource type and owner between the 2014 model and reality. The non-conventional renewable energy category comprises wind and 'other' generation, which itself includes mini-hydro, biomass, and solar-PV. The model does an excellent job of capturing seasonal large hydro patterns; however, monthly fuel oil, coal, and diesel generation differs between the model and reality depending on when plants experience forced outages. Actual generation data suggests that Sri Lanka experienced coal outages in January, February, and August of 2014, whereas in the model, available energy from coal is lowest in February and May. Wind generation is $11.5 \%$ higher in the model than reality, which we determined to be caused by discrepancies in wind farm commission dates.

It is important to remember that although the 2014 model does a good job of capturing annual and monthly generation patterns, it does not attempt to mimic the exact hour-by-hour 2014 dispatch. Because of the inherent uncertainty in future-year system operations, a model overly calibrated to the unique events of 2014 would provide no advantage when expanded to 2025 . 

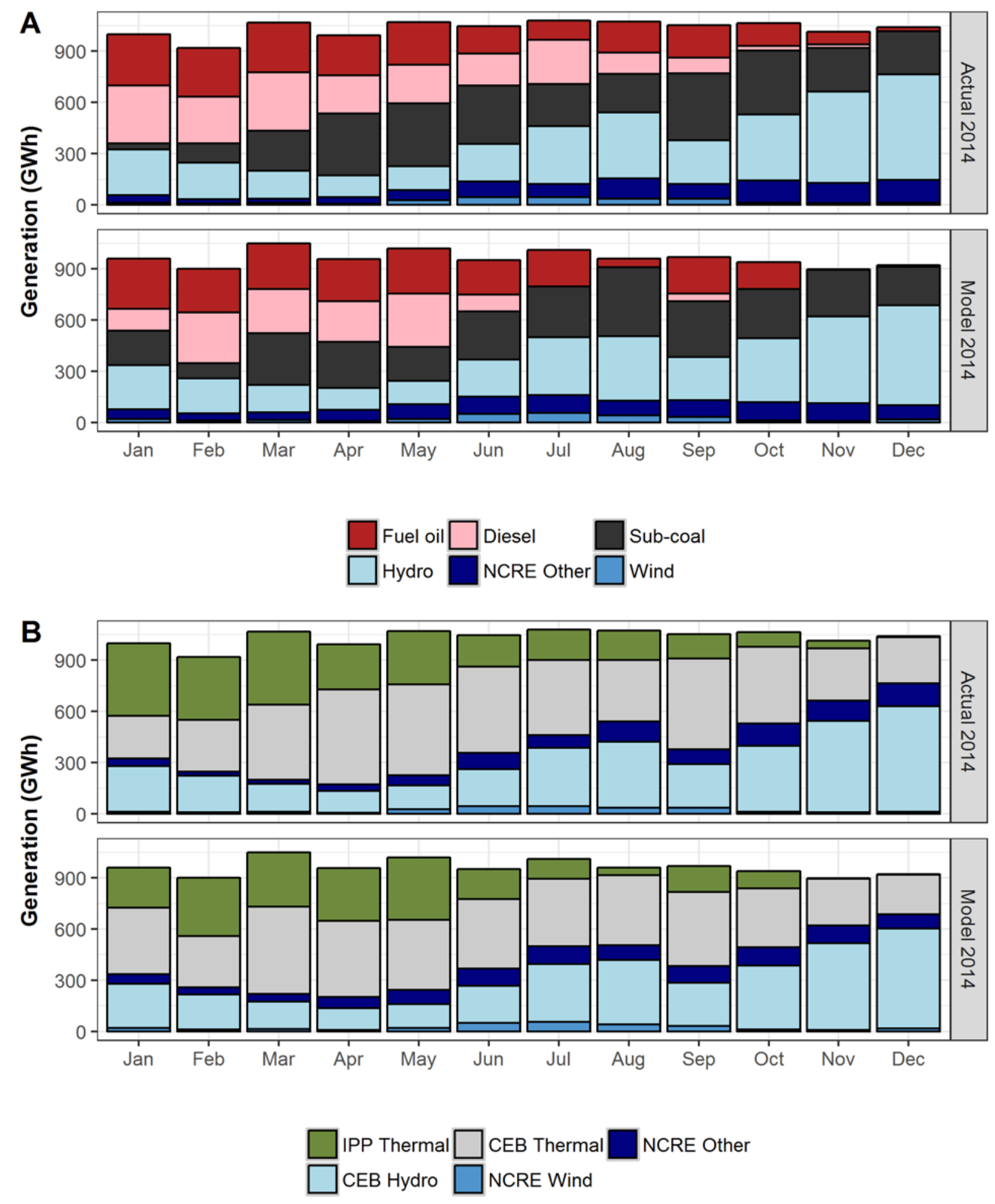

Figure 23. Comparison of Sri Lanka's actual and modeled 2014 generation by (A) resource and (B) ownership 
Figure 24 provides a closer look at the monthly behavior of CEB's large hydro between the 2014 model and reality. Setting monthly maximum energy limits (for reasons discussed in section 2.3.2) ensures that modeled 2014 large hydro generation never exceeds actual output. To the extent that available zero-cost hydro energy remains unused (the difference between the blue and red bars), we can assume that a combination of reservoir limitations, inflow constraints, thermal fleet inflexibility, and start costs prevent its dispatch.

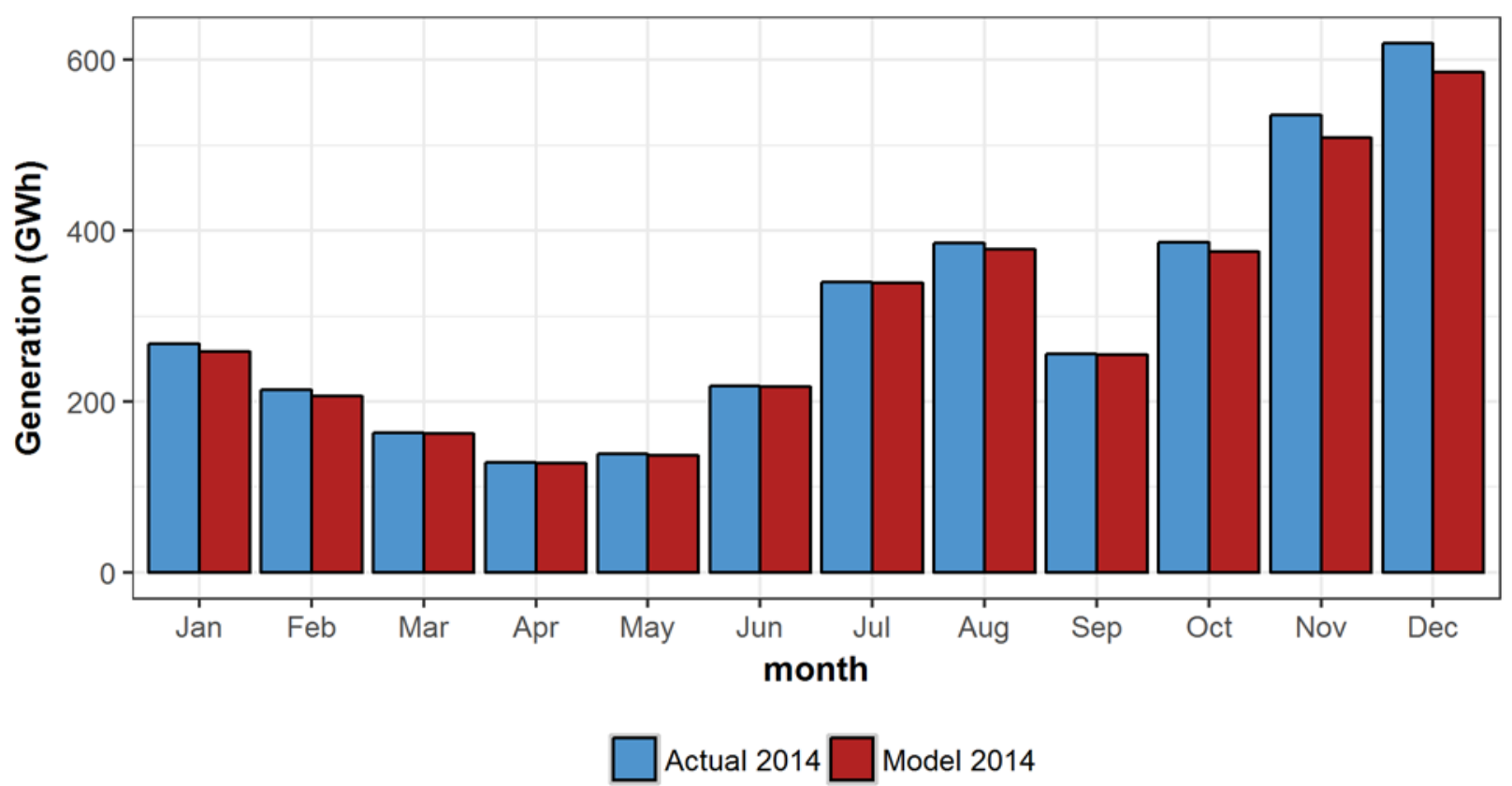

Figure 24. Comparison of Sri Lanka's actual and modeled 2014 CEB-owned hydro generation

A core portion of the 2014 validation was the examination of differences between 2014 modeled and actual thermal generation at the plant level. Large substitutions in generation by plant would suggest that either the model uses inaccurate or incomplete cost data or that in real operations the system does not follow a strictly least-cost dispatch. Either effect might reduce the 2025 model's ability to predict the impacts of sensitivities on future system operations. Figure 25 compares actual and modeled 2014 generation at six of Sri Lanka's major thermal installations. Because there is no consistent substitution of one plant for another, the expanded 2025 model likely does an adequate job of capturing CEB's least cost dispatch. 

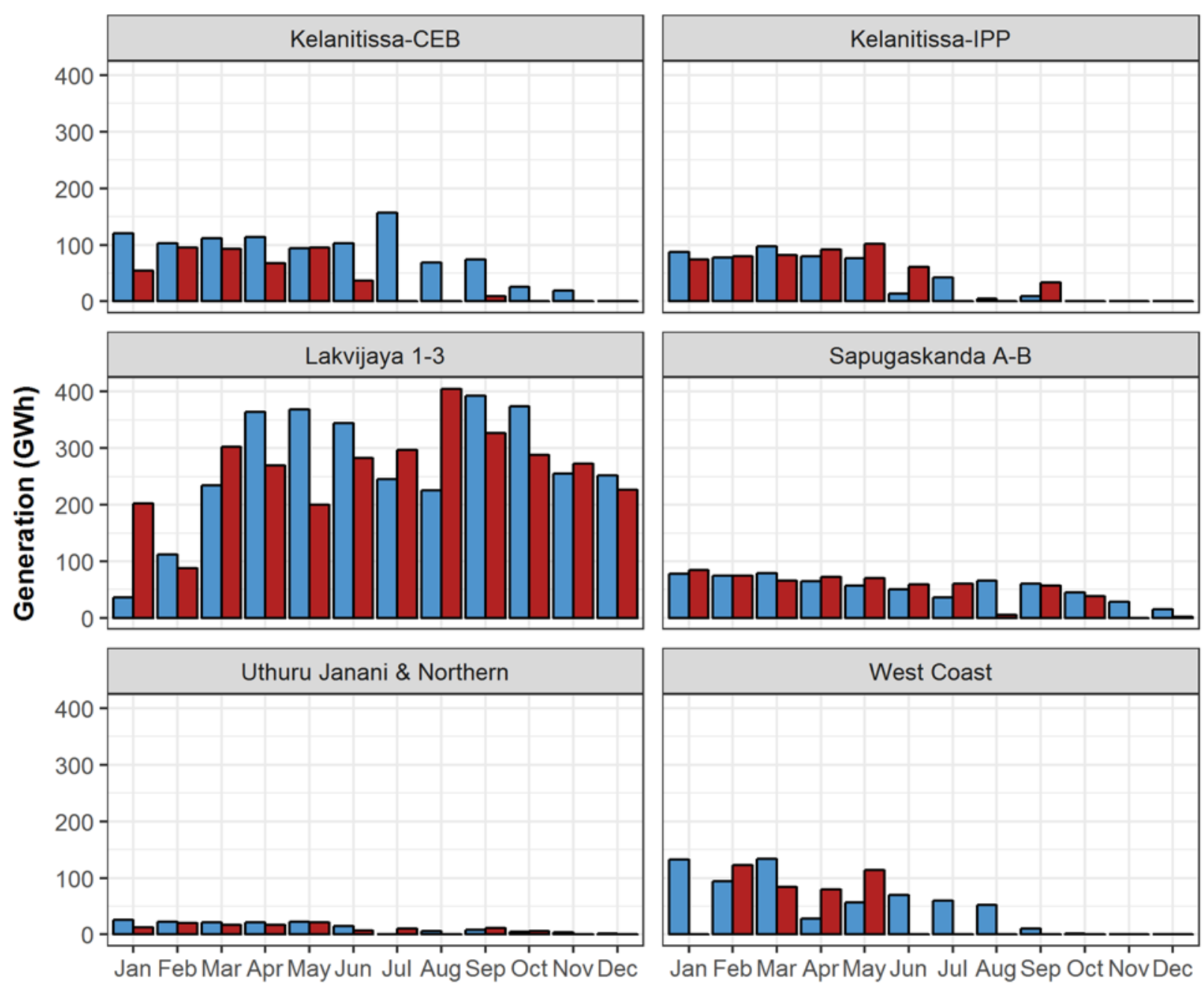

month

Actual $2014 \square$ Model 2014

Figure 25. Comparison of actual and modeled 2014 monthly generation at six of Sri Lanka's major thermal installations

The 2014 model experiences $480 \mathrm{MWh}$ of unserved energy over the course of the second half of February. The unserved energy results from seasonally low hydro availability, and a lack of coal, because the second and third Lakvijaya units had not yet commissioned and the first unit undergoes scheduled maintenance. Unmet reserves total $7.65 \mathrm{GWh}$ and cluster in the second half of February. 